\begin{tabular}{|l|l|l|l|}
\hline Inv. Pesq. & 28 & Pógs. 173-209 & abril 1965 \\
\hline
\end{tabular}

\title{
Hidrografía y fitoplancton de las costas de Castellón, de agosto de 1962 a julio de 1963
}

\author{
$1^{30}$ \\ FELIPE MITOZ* Y JOSE M. SAN FETSU*
}

\section{NTOT)LOCION}

Los datos del presente estudio se obtuvieron todos en la estación B, ya citada en trabajos anteriores de esta serie, cuyas características repetimos : posición $39^{\circ} 55^{\prime} 30^{\prime \prime} \mathrm{N}$ y $00^{\circ} 21^{\prime} 30^{\prime \prime} \mathrm{E}$; profundidad, $78 \mathrm{~m}$; y distancia a la costa 16,8 millas. l)ichos datos proceden de 12 salidas distribuidas algo irregularmente a lo largo del periodo de observaciones.

Al igual que en estudios anteriores, las determinaciones se hicieron con barco fondeado, anotando los siguientes datos: a) temperatura del aire, b) presión atmosférica, c) dirección del viento, $d$ ) intensidad aproximada del mismo, $e$ ) clase de nubes, $f$ ) nubosidad estimada en \%, g) visibilidad, $h$ ) estado del mar, $i)$ temperatura de aguas superficiales, j) profundidad de visión del disco de Secchi, $k$ ) dirección de la corriente y velocidad de la misma mediante flotador de tipo sifonóforo y l) perfiles térmicos medios con termistor. Se tomaron muestras de agua para la determinación de plancton sedimentado, fósforo inorgánico disuelto y salinidad, a profundioades de $0,5,10,20,30,50$ y $75 \mathrm{~m}$, anotándose también sus correspondientes temperaturas; también se tomaron otras muestras de agua a $0,10,20,40$ y $60 \mathrm{~m}$ para separación de fitoplancton mediante filtración a travís de papel de filtro Albet 242 y extracción de sus pigmentos con acetona al $90 \%$. A continuación se llevaban a caho pescas verticales de zooplancton desde las proximidades del fondo hasta la superficie con una red de $0,26 \mathrm{~m}^{2}$ de boca, pescas horizontales

* Laboratorio del Inst. de Invest. Pesqueras. Monturiol, 2. Grao - (Asteldón. 
de fitoplancton (Zurich, núm. $18 \mathrm{xx}$ ) y otras horizontales de zooplancton a $0,10,20,40$ y $60 \mathrm{~m}$ (Zurich, núm. $5 \mathrm{xx}$ ). Los detalles sobre técnicas de trabajo se han descrito o citado en publicaciones anteriores de esta serie y especialmente hacemos referencia a Herrera \& Margalef (1961).

Manifestamos nuestro pesar porque dificultades ajenas a nuestra voluntad nos impidieron, en algunos casos, distribuir con más regularidad las tomas de muestras y en otros, efectuar todas las operaciones dispuestas para cada salida, lo cual dificulta precisar o matizar el estudio correspondiente a este interesantísimo ciclo anual.

A través de los datos numéricos obtenidos directamente o elaborados, como se expresa en las correspondientes tablas, trataremos en los capitulos siguientes de estudiar, lo más completamente posible, el presente ciclo hidrobiológico, sustancialmente básico para el conocimiento pesquero de esta zona y además lo relacionaremos con los ciclos anuales anteriores.

Con el presente ciclo anual parece que hemos debido de completar el estudio de un macrociclo. A la abundancia de fitoplancton del ciclo 1950-1951, juzgada solamente a través del color de sus extractos de pigmentos y algún otro dato complementario, siguen los ciclos siguientes que aunque con diversas alternativas, van marcando una lenta disminución de la productividad biológica hasta desembocar en el presente en el que, de nuevo, la producción se eleva hasta térıinos comparables a la del primitivo ciclo 1950-1951, sin embargo el actual, mucho mejor estudiado, nos permitirá establecer con más precisión sus características. ¿Estamos en presencia de macrociclos de unos diez años de duración? La existencia de ciclos plurianuales parece probable aunque, por ahora, nada se pueda decir en cuanto a su duración ni en cuanto a la regularidad con que se sucedan. La continuación de estos estudios en años sucesivos nos proporcionará material para un conocimiento más preciso de los fenómenos relacionados con la fertilidad del mar.

Los estudios sobre contenido, distribución, etc., del zooplancton correspondientes a este ciclo serán publicados en comunicación aparte por otro autor. A través de estas líneas queremos mostrar nuestro agradecimiento al Sr. Sanjuan, por poner a nuestra disposición ciertos datos meteorológicos de particular interés, también agradecemos la colaboración eficaz de nuestro patrón de pesca, Sr. Canales, y de la Srta. Martín que nos ayudó en algunos de los trabajos materiales y cálculos realizados.

\section{METEOROLOGTA E HIDROGRAFIA}

Vientos. Fn la tabla I se presentan los valores medios de la intensidad de viento en la zona pesquera del Grao de Castellón durante el 
presente ciclo anual. En dicha tabla aparecen distribuidos, como en trabajos anteriores, por periodos de tiempo de diez días, dando igualmente los valores de la resultante total $y$ de la componente favorable.

$$
\text { T A B L A I }
$$

Valores medios decenales de la intensidad de los vientos (en $\mathrm{kg} / \mathrm{m}^{2}$ ) en relación con el afloramiento.

\begin{tabular}{|c|c|c|c|c|c|}
\hline \multirow[b]{2}{*}{ DECENA } & \multicolumn{2}{|c|}{ INTENSIDAD DE LOS VIENTOS } & \multirow[b]{2}{*}{ Decena } & \multicolumn{2}{|c|}{ INTENSIDAD DE LOS VIENTO } \\
\hline & $\begin{array}{l}\text { RESULTAN- } \\
\text { TE TOTAT, } \\
\text { (1) }\end{array}$ & $\begin{array}{c}\text { COMPONENTE } \\
\text { FAVORABLE } \\
(2)\end{array}$ & & $\begin{array}{l}\text { RESULTAN- } \\
\text { TE TOTAL } \\
\text { (1) }\end{array}$ & $\begin{array}{c}\text { COMPONENTF } \\
\text { FAVORABLE } \\
\text { (2) }\end{array}$ \\
\hline 1962 & & & 1963 & & \\
\hline 1. a julio & $-0,467$ & 0,002 & 1. enero & $+0,246$ & 0,329 \\
\hline $2.8 \gg$ & $-0,253$ & 0,023 & $2 x^{\mathrm{a}} \gg$ & $-0,232$ & 0,062 \\
\hline $3 . \mathrm{a} \gg$ & $-0,426$ & 0,002 & 3. & $-0,870$ & 0,049 \\
\hline 1. agosto & $-0,741$ & 0,048 & 1. a febrero & $-0,043$ & 0,086 \\
\hline $2 .{ }^{2} \quad 2$ & $-0,259$ & 0,018 & $2 .^{3} \quad \gg$ & $+0,579$ & 0,579 \\
\hline $3 .{ }^{a} \quad \gg$ & $-0,084$ & 0,023 & $3 .^{\mathrm{a}} \quad 》$ & $-0,157$ & 0,060 \\
\hline 1. a septbre. & $-0,171$ & 0,028 & 1. a marzo & $+0,158$ & 0,196 \\
\hline $2.3 \gg$ & $-0,498$ & 0,002 & $2{ }^{2} \quad 》$ & $+0,431$ & 0,482 \\
\hline $8 . \mathbf{a} \quad \gg$ & $-0,598$ & 0,038 & $3 .^{a} \gg$ & $+0,109$ & 0,280 \\
\hline 1. a octubre & $-0,253$ & 0,000 & 1. a abril & $+0,157$ & 0,223 \\
\hline $2 .^{\mathrm{a}} \gg$ & $-0,930$ & 0,063 & $2 .{ }^{\mathrm{a}} \gg$ & $+0,019$ & 0,159 \\
\hline 3. ${ }^{\mathbf{2}} \gg$ & $-0,056$ & 0,006 & $3 .^{\mathbf{a}} \quad \square$ & $-0,203$ & 0,010 \\
\hline 1. a ncvbre. & $+0,051$ & 0,199 & 1. a mayo & 0,046 & 0,008 \\
\hline $2 .^{a} \gg$ & $+0,091$ & 0,126 & $2 .^{a} \gg$ & $+0,029$ & 0,085 \\
\hline $\mathbf{3} . \mathbf{a} \quad \gg$ & -0.027 & 0,084 & $3 .^{a} \gg$ & $-0,276$ & 0,084 \\
\hline 1. a dicbre. & $-0,052$ & 0,003 & 1.* junio & $+0,022$ & 0,279 \\
\hline $2 .^{a} \gg$ & $+0,189$ & 0,184 & $2 a^{a} \gg$ & $\begin{array}{l}0,450 \\
\end{array}$ & 0,014 \\
\hline $3{ }^{a}$ & $+0,127$ & 0,127 & $\mathbf{3}^{\mathrm{a}} \gg$ & $-0,175$ & 0,015 \\
\hline
\end{tabular}

(1) Calculada según la expresión $(1 / n) \Sigma k_{\theta} v_{e}$, en la que $v_{\theta}$ son las intensidades observadas; $n$, el número de observaciones, y $k_{s}$ un coeficiente dependiente de la dirección, que vale 1 para la dirección $\mathrm{SW}, 0,7$ para $\mathrm{S}$ y $W, 0$ para $\mathrm{NW}$ y $\mathrm{SE}$, $-0,7$ para $\mathrm{N}$ y $\mathrm{E}, \mathrm{y}-1$ para $\mathrm{NE}$.

(2) Calculada como en el caso anterior, pero igualando a o los valores negativos de $k_{\ell}$.

Si consideramos los valores observados en el presente ciclo anual, a través de la componente favorable, durante los meses considerados como productivos : noviembre-abril, con los de los restantes ciclos anuales estudiados, veremos que el actual es uno de los que presentan mejores características desde el punto de vista del afloramiento de aguas, siendo superado solamente por el ciclo 1957-1958 como puede observarse en la tabla II. En el ciclo anual que estudiamos, todos los meses presentan alguna decena de días con predominio de los vientos favorables al afloramiento, pero es notable el completo predominio en el mes de marzo que, ininterrumpidamente se prolonga hasta la última decena de abril. 
T A B I,

Bistribución de vientos en

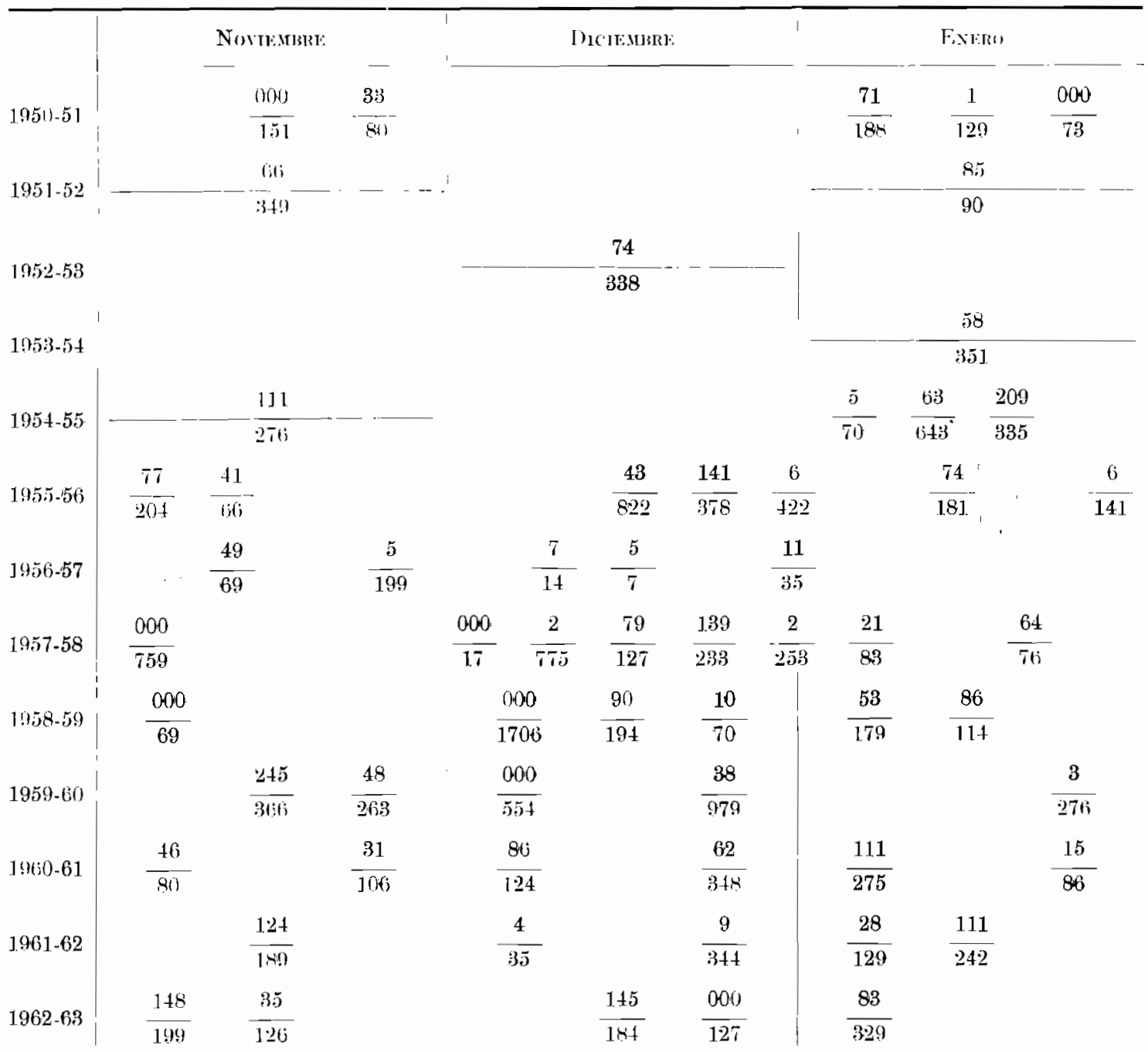

La distribución de los vientos se presenta por meses, decenas o semanas, según los ciclos anuales, y viene representada por un quebrado constituido por los vientos desfavorables al afloramiento en el numerador y favorables en el denominador, am. 
I I

semestre production

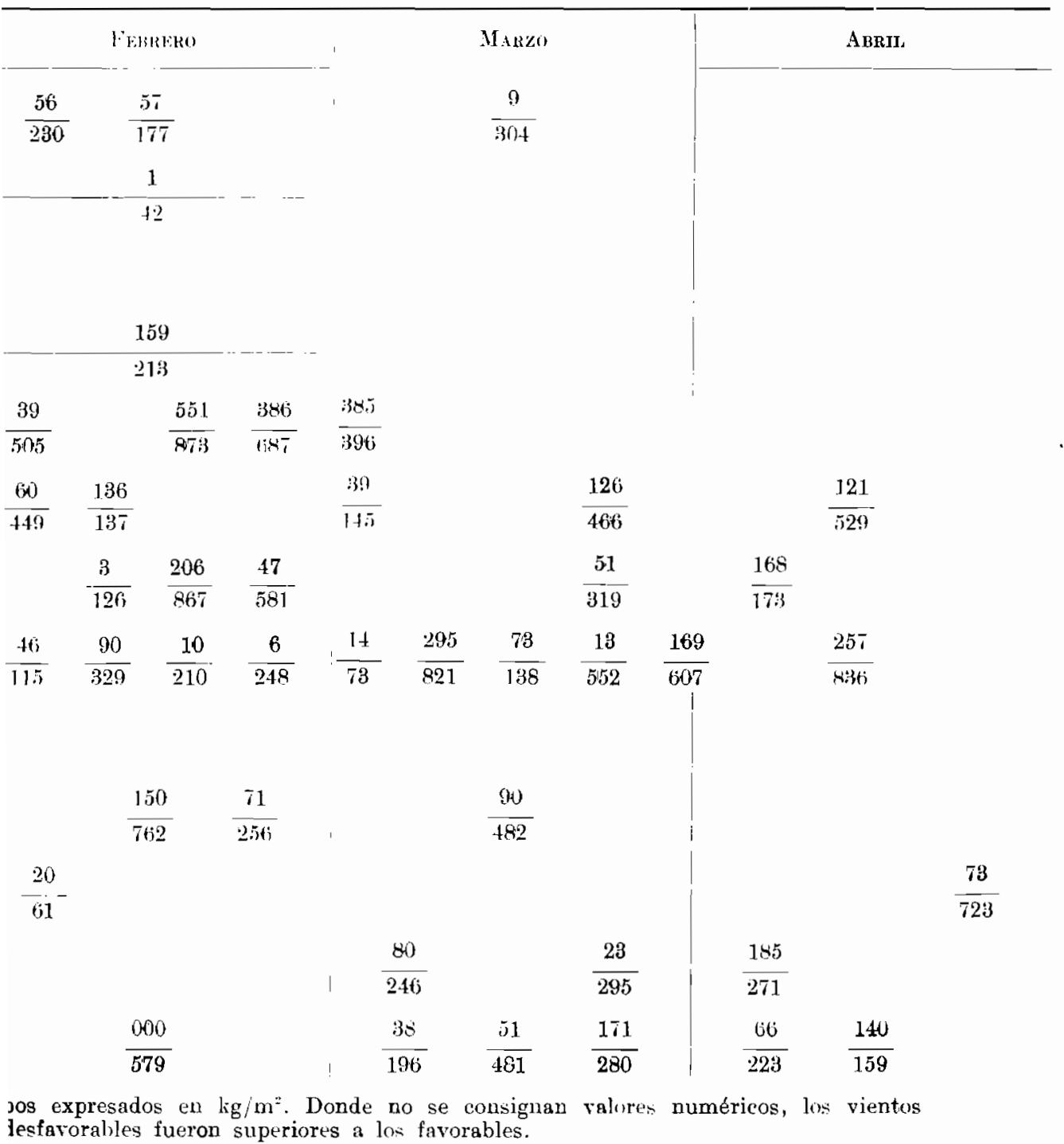


Temperatura y salinidad. En la figura 1 se representa el ciclo anual de temperaturas. En ella se obserra que a partir de diciembre y hasta finales de abril aparece una homogeneidad de temperaturas a todas las profundidades, lo que permitió una mezcla vertical de las aguas. Antes y después de este periodo aparece la clásica termoclina estival. Lıo más notable que se observa en la figura es la aparición en los meses de febrero y inarzo de una capa superficial algo más fría que el resto de la masa. Al comparar con años anteriores, observamos que, en gencral, toda la masa de agua en dichos meses es más fria, hecho que se prolonga hasta
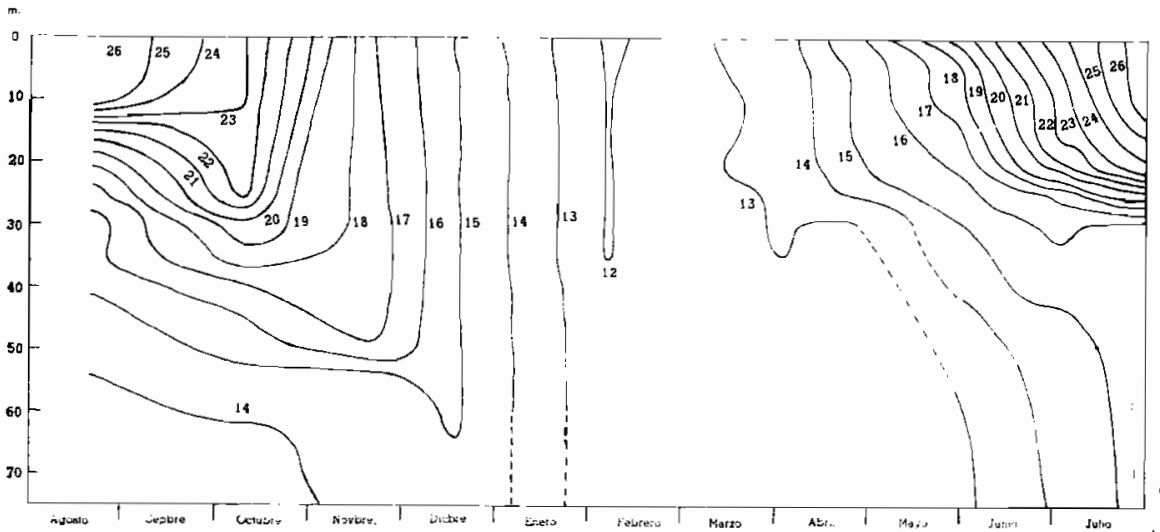

Fia. 1. - Isopletas de la distribución de teinperaturas en la estación B, de agosto de 1962 a julio de 1963. A la izquierda escala de profundidades.

el mes de abril, mayo y junio, especialmente en el londo, como se puede comprobar en la tabla IIT. Parece como si el flujo ordinario de las aguas hubiera sido desplazado por otras masas de agua de procedencia diferente, más fría, probablemente del fondo.

La salinidad, en el presente ciclo anual (fig. 2), en consonancia con la disminución térmica, experimentó un incremento considerable a partir de las determinaciones de febrero, permaneciendo por encima de $38 \% 0$ en el mes de marzo y parte de abril en todas las profundidades, y conservando dichos valores las aguas intermedias y profundas hasta el mes de julio. Queremos resaltar que el 25 de abril se obtuvo el mayor valor de los registrados por nosotros en estas aguas: $38,28 \%$. Fn años anteriores sólo se llegó a alcanzar el valor 38,21\%o en febrero de 1957. En relación con ciclos anuales anteriores solamente el de 1956-1957 es comparable con el presente, si bien aquél no aventajó a éste, ya que a pesar de comenzar más temprano casi siempre los valores permanecieron por debajo de los del actual. También es digno de notar que la elevación de la salinidad se presenta en dos ocasiones diferentes, la primera a partir de febrero y la segunda en mayo. 
T A B L A I I I

Diferencia de temperatura del año 1963 a los anteriores

\begin{tabular}{|c|c|c|c|c|c|c|c|c|c|c|}
\hline & \multicolumn{3}{|c|}{1959} & \multicolumn{7}{|c|}{1960} \\
\hline & 6-IV & 21-IV & $26-\mathrm{V}$ & 4-II & 26.II & 8-III & 5.IV & 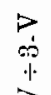 & $\begin{array}{l}\vec{z} \\
=\overrightarrow{0}\end{array}$ & $N^{3}$ \\
\hline $19633=$ & 3.IV & 25-IT & $30 . \mathrm{V}$ & 6-II & 2.2 -II & $15-\mathrm{III}$ & 3-IV & $\begin{array}{l}3 \\
\dot{0}\end{array}$ & 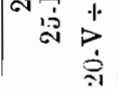 & ${ }^{N} \dot{p}$ \\
\hline
\end{tabular}

\begin{tabular}{|c|c|c|c|c|c|c|c|c|c|}
\hline 0 & $-2,0$ & $+0,4$ & +11 & -1.25 & -105 & -1.15 & & & \\
\hline 5 & $-2,3$ & $\begin{array}{l}+v, 4 \\
+0,45\end{array}$ & $\begin{array}{r}0.1 \\
0.25\end{array}$ & $\begin{array}{l}-100 \\
-120\end{array}$ & $-0,60$ & $-1,10$ & $\begin{array}{r}-1,90 \\
-0,90\end{array}$ & +0.10 & $\begin{array}{r}-2.9 \\
-295\end{array}$ \\
\hline 10 & $-1,1$ & $+0,35$ & & -1.15 & $-0,30$ & $-0,65$ & $-0,95$ & $+0,20$ & $\begin{array}{r}-2,00 \\
\end{array}$ \\
\hline 15 & & & & -1.15 & & & $-0,85$ & $+0, \mathbf{2 5}$ & $-1,60$ \\
\hline 20 & $-0,4$ & $+0,25$ & $-0,6$ & $-1,1 ;$ & $-0,20$ & $-0,50$ & $-0,55$ & $-0,95$ & $--0,95$ \\
\hline 25 & & & & -1.15 & & & $-0,55$ & $-1,60$ & $-0,0 \mathrm{~s}$ \\
\hline 30 & $-0,9$ & $-1,70$ & $+0,1$ & $-\mathrm{L}, 15$ & $-0,50$ & $-0,15$ & $-0,25$ & $-1,70$ & -0.35 \\
\hline 35 & & & & $-1,15$ & & & $-0,10$ & $-0,90$ & - $0, \mathbf{2 5}$ \\
\hline 40 & $-0,95$ & & & $-0,95$ & & & $-0,15$ & $-0,95$ & $-0,60$ \\
\hline $4 \bar{J}$ & & & & $-0,95$ & & & $-0,1 \cap$ & $-0,65$ & $-0,60$ \\
\hline 50 & -0.95 & & $-0,45$ & $-0,90$ & $-0,05$ & $+0,60$ & $-0,05$ & $-0, \tilde{5} \overline{5}$ & $-0,95$ \\
\hline 55 & & & & —1,90 & & & $-0,15$ & $-0,50$ & $-1,10$ \\
\hline 60 & & & & $-0,90$ & & & $-0,15$ & $-0,50$ & \\
\hline $\begin{array}{l}65 \\
70\end{array}$ & $-0,20$ & & & & & & $\begin{array}{l}-0,15 \\
-0,15\end{array}$ & & \\
\hline 75 & & & & & $-0.1 \%$ & -0.25 & $-0,15$ & $-0,50$ & $-0,65$ \\
\hline $\begin{array}{l}\text { Media } \\
\text { Media }\end{array}$ & $\begin{array}{l}-0,95 \\
\text { anual }\end{array}$ & $\begin{array}{l}-0,05 \\
-0,35\end{array}$ & $-0,00$ & $-1,05$ & $-0,40$ & $-0,40$ & $\begin{array}{l}-0,45 \\
-0,65\end{array}$ & $-0,6 \check{\beth}$ & $-1,10$ \\
\hline & & & 1961 & & & & 19 & & \\
\hline & 7-II & 27-II & 22-III & $25 . V$ & & $17-\mathrm{II}$ & 7.III & 27-III & $12-\mathrm{IV}$ \\
\hline 1963 & $=6-\mathrm{II}$ & $22-\mathrm{II}$ & 15-III & $30 . \mathrm{V}$ & & 22.II & 15-III & 3-IV & 3-IV \\
\hline $\mathrm{m}$ & & & & & & & & & \\
\hline 0 & $-0,75$ & $-1,10$ & $-0,90$ & $-1,0$ & & $-1,20$ & $+0,70$ & $+0,30$ & $-0,90$ \\
\hline 5 & $-0,70$ & -1.00 & $-1,10$ & $-2,55$ & & $-0,90$ & $+0,50$ & & $-0,80$ \\
\hline 10 & $-0,65$ & -0.70 & $-1,20$ & $-2,60$ & & $-0,90$ & $+0,40$ & $+0,05$ & $-0,90$ \\
\hline 15 & $-0,75$ & & & $-3,50$ & & & & $+0,05$ & $-0,90$ \\
\hline 20 & $-1,05$ & $-0,60$ & $-0,70$ & $-2,50$ & & $-0,80$ & $+0,40$ & $+0,15$ & $-0,80$ \\
\hline 25 & $-1,05$ & & & $-0,60$ & & & & $-0,05$ & $-0,95$ \\
\hline 30 & $-1,05$ & $\square_{0,35}$ & $-0,45$ & $-0,10$ & & $-0,9 \check{\dot{u}}$ & $-0,6^{-}$ & $-0,05$ & $-0,95$ \\
\hline 35 & $-1,05$ & & & $+0,10$ & & & & $-0,10$ & $-1,00$ \\
\hline 40 & $-0,85$ & & & $-0,10$ & & & & $-0,15$ & $-1,05$ \\
\hline 45 & $-0,85$ & & & $-0,25$ & & & & $-0,20$ & $-0,05$ \\
\hline 50 & $-0,80$ & $-0,60$ & & $-0,45$ & & $-1,00$ & & $-0,25$ & $-0,45$ \\
\hline 55 & $-0,80$ & & & $-0,60$ & & & & $-0,25$ & -0.25 \\
\hline 60 & $-0,80$ & & & & & & & $-0,25$ & $-0,15$ \\
\hline 65 & & & & & & & & $-0,45$ & $+0,05$ \\
\hline 70 & & & & & & & & $-0,45$ & $+0,05$ \\
\hline 75 & & $-0,50$ & $+0,05$ & $-0,55$ & & $-0,45$ & $-0,35$ & $-0,45$ & $+0,05$ \\
\hline $\begin{array}{l}\text { Media } \\
\text { Media }\end{array}$ & $\begin{array}{c}\text {-0,85 } \\
\text { anual }\end{array}$ & $-0,70$ & $-0, \overline{85}^{0,70}$ & $--1,15$ & & -0.90 & $+0,1 \ddot{c}$ & $\rightarrow_{35}^{0,15}$ & $-0,55$ \\
\hline
\end{tabular}


m.

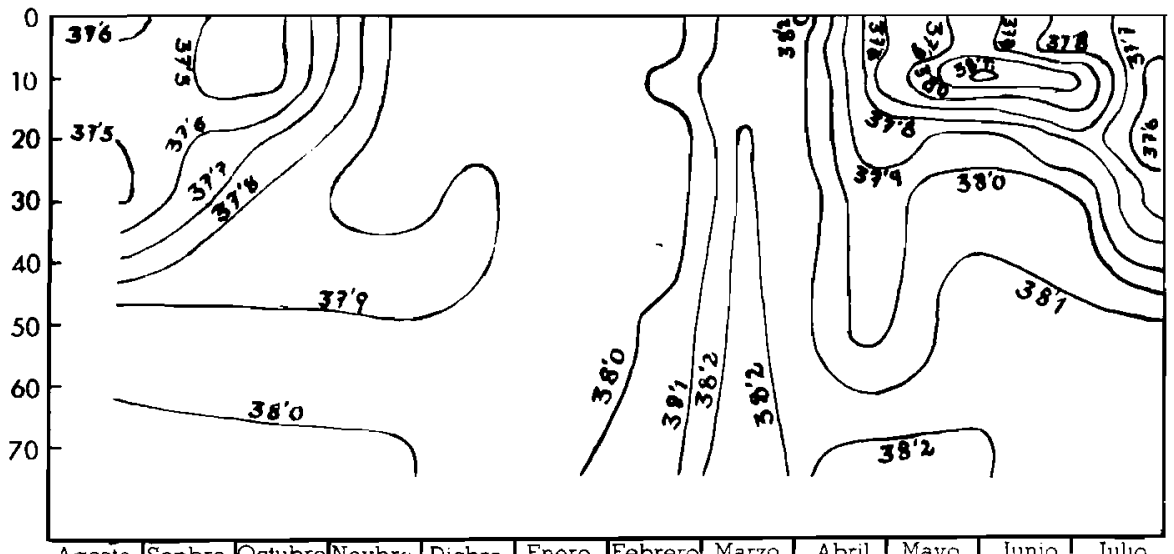

Fig. 2. - Isopletas de la distribución de salinidades en la estación B, de agosto de 1962 a julio de 1963. A la izruierda escala de profundidades.

Como puede observarse en la figura 3 , la densidad del agua, a partir de febrero, se eleva considerablemente sobrepasando ampliamente el valor 28,5 durante éste y los restantes meses siguientes en todas las profundidades. Solamente a finales de abril empieza a disminuir en las capas superiores aunque en las capas más profundas se llega a alcanzar el valor máximo: 29.0. En los meses siguientes persisten los valores altos de $\sigma_{t}$ cerca del fondo.

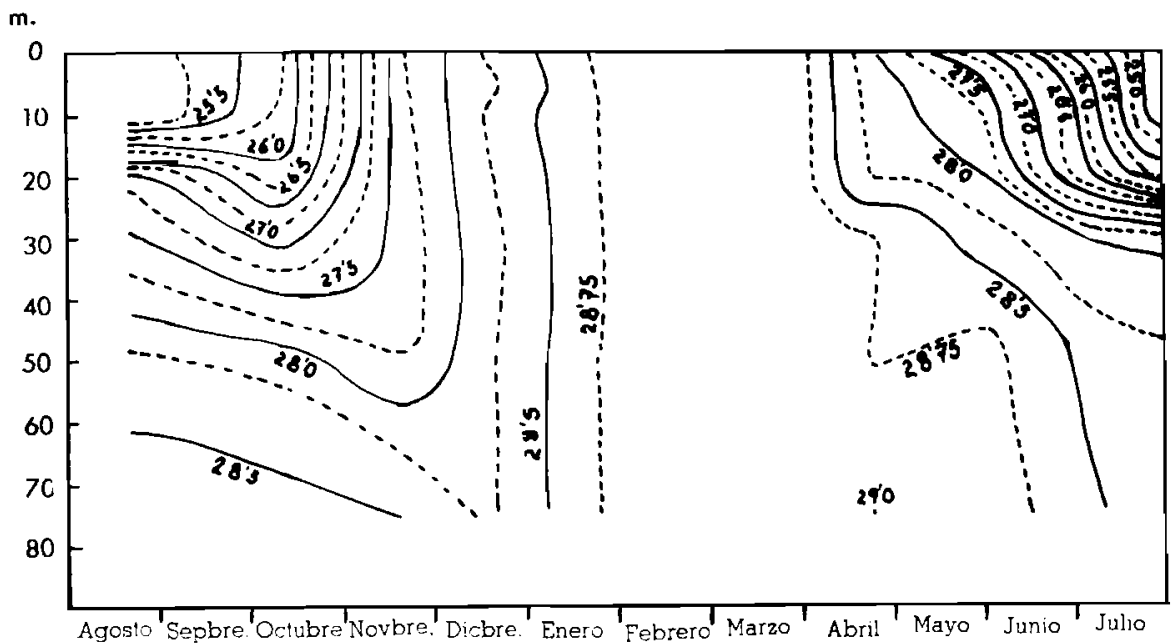

Fig. 3. - Isopletas de la distribución de valores $\sigma_{t}$ en la estación B, de agosto de 1962 a julio de 1963. A la izquierda escala de profindidades. 
Sales mutrientes. ('omo en ciclos anteriores hemos seguido caracterizando la capacidad nutriente de las aguas a través de su concentración de fosfatos inorgánicos disueltos. Hin la primera parte dol ciclo los ralores caen dentro de lo normal en relación con ciclos anteriores para iguales fechas, del orden de 0,3-0,5 $\mu \mathrm{g}$ at./l (fig. 4). Fn la mimera determinación de febrero se observa ya un incremento, llegando a alcanzar: elevados valores en el mes de marzo, desciende paulatinamente en ahril y de nuevo ruelve a observarse $u 1$ máximo en maro, annque inferior m.

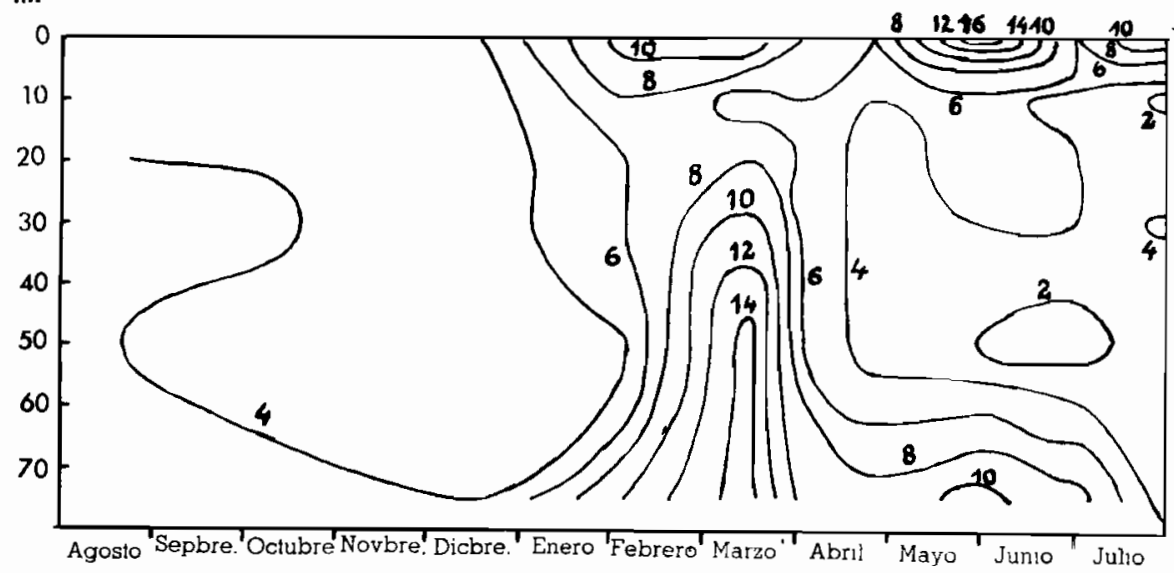

Fici. 4. - Distribución del fosfato inorgánico en $\mu \mathrm{g}$-at. $\mathrm{P} \cdot 1 \mathrm{u}^{*} / 1$ m la estación B. de agosto de 1962 a julio de 1963 . A la izquierda escala de profundidades. Cada unidad de las usadas en la figura equivalen a 0,01 ig-at. P/l y aproximadomente a $0.3 \mathrm{mg} P / \mathrm{m}^{3}$.

al anterior. En relación a ciclos anuales pasidor podemos comprobar que durante los meses de febrero a mayo, la cantidad de fósforo inorgánico disuelto lue aproximadamente el doble que el ralor medio interanual.

Transparencia y corrientes. La transparencia se media con el disco de Secchi, como se ha venido haciendo hasta el presente. En este ciclo anual la penetración de la luz en el agua del mar se comporta de una manera análoga a lo ocurrido en otros ciclos anuales, aunque es de destacar un valor mínimo de $7,3 \mathrm{~m}$ (fig. 5) en la determinación de marzo que supone el valor más bajo registrado en esta estación hasta la fecha.

El estudio de la corriente superficial nos muestra que, solamente en dos ocasiones, el 15 de marzo $r$ el :3 de abril, aparecieron flujos de agua con dirección hacia alta nar, en lás demás ocasiones las corrientes solian ir aproximadauente paralelas a la costa o perpendicularmente hacia ella. 


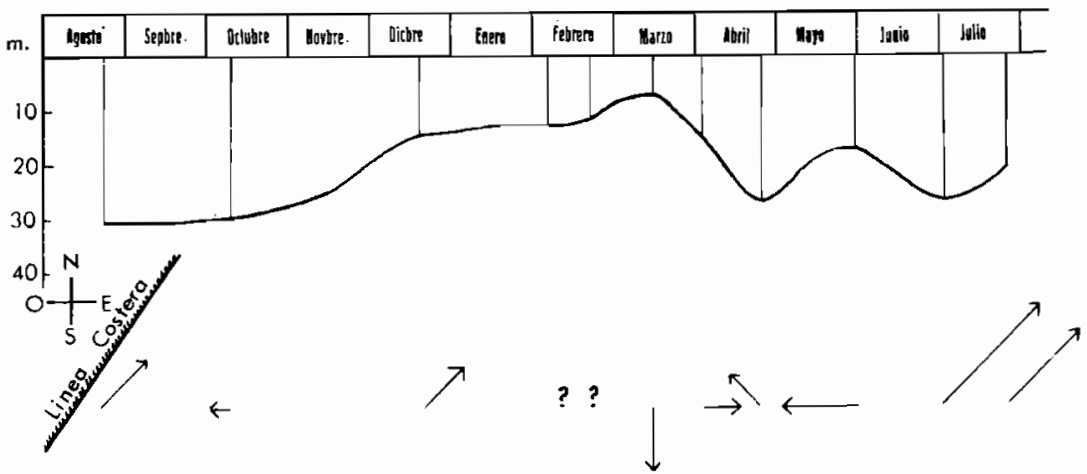

Fig. 5. - Transparencia del agua en la estación B, de agosto de 1962 a julio de 1963, expresada por la profundidad de visión del disco de Secchi. A la izquierda, escala do profundidades en metros. En la parte baja, dirección y velocidad en centímetros/segundo de las corrientes superficiales.

Interpretación de los datos. De todo lo expuesto anteriormente se deduce que el presente ciclo anual ha tenido unas características bien definidas que le distinguen de todos los estudiados anteriormente : vientos favorables al afloramiento ahundantes, temperaturas muy bajas en el tiempo apropiado, altas salinidades y elevada concentración de sales nutrientes. A primera vista parece evidente la existencia de un marcado afloramiento. Ultimamente MARrialeF (1963) y Margalef \& Herrera (1964) dudan de la primitiva idea que se tenía sobre la existencia de afloramientos originados por efectos del viento. Actualmente, después de trece años de estudios consecutivos de las relaciones entre la intensidad de vientos favorables y producción, nos encontramos con más elementos de juicio para poder juzgar el fenómeno.

En efecto, el presente ciclo anual aparece, a juzgar por los datos de fitoplancton, sales nutrientes y salinidad, como el más productivo de todos los últimamente estudiados, si desestimamos el de 1950-1951 que solamente pudo ser caracterizado a través de la concentración de fitoplancton y algún otro dato, sin embargo el efecto favorable del viento en el presente ciclo, si bien es muy elevado, no parece superior al de 1957-1958 (tabla II) en el cual no se observaron salinidades superiores a $38 \%$ ni concentraciones de fósforo inorgánico disuelto superiores a los valores interanuales para el semestre productivo, sino más bien inferiores. Altas salinidades se observaron en el ciclo 1956-1957 (Herrfra \& MargaleF, 1957), aunque no tanto como en el actual, mientras que las concentraciones de fósforo eran más bien bajas y el efecto de los vientos favorables al afloramiento escaso, excepto en el mes de febrero. Las concentraciones de fósforo mís elevadas, dentro del tono medio de los ciclos 
estudiados, se dan en el ciclo 1960-1961 (Herrera \& Margalef, 1963), sin embargo, la salinidad no alcanza el valor $38 \%$ excepto al comienzo del ciclo productivo y el efecto del viento no pudo tener significativa importancia. No alargaremos más esta relación, ya que por otra parte hemos descrito lo inás notorio.

Aunque el efecto del viento favorable al afloramiento, dentro de las limitaciones de continuidad, fuerza, dirección, altul'al de elevación de aguas profundas, condiciones de la costa, etc., es estinuado por Yosida (1955) que debe persistir por más de una semana. parece que deben ser tenidos en cuenta otros factores para que el efecto físico de remnción de las aguas rinda sus frutos de producción biológica. Es preciso que las aguas elevadas a la zona fótica tengan previamente su correspondiente carga de sales nutrientes. En nuestro caso y según datos inéditos, la remoción de menos de 7 cull de la película superficial del sedimento es suficiente para alcanzar una concentración teórica de fósforo disuelto, a lo largo de toda la columna de agua, igual al máximo valor registrado. Esto nos hace pensar que el efecto del viento favorable al afloramiento en esta zona debe ser, aún en los ciclos anuales más propicios, insufi-. ciente para un abonado eficaz de las aguas, como ocurrió en el citado ciclo 1957-1958.

Descartado el efecto del viento cumo agente principal del abonado marino, hemos de pensar en otros, como puede ser el de las corrientes profundas, con lo que en cierto modo puede explicarse el sistemático descenso de la salinidad y producción a partir del ciclo anual 1956-1957, a pesar de las diversas alternativas de los efectos del viento en los últimos años, va que también sistemáticamente ha venido disminuvendo a partir de aquel ciclo la influencia de las corrientes a juzgar por los valores de salinidad últimamente alcanzados.

Los estudios sobre corrientes superficiales en nuestra zona (SUAU \& Vives, 1958 y Bougis, 1958) ponen de manifiesto la tendencia general durante la mayor parte del año a seguir la dirección NE-SW, aunque también se han señalado otras direcciones en determinadas épocas del año. El estudio de Furnestin (1960), sobre dirección de corrientes en profundidad en esta zona, es interesante, aunque se realizó en un período muy limitado de tiempo y en la época menos apropiada para su aplicación a nuestro estudio (14 junio-20 julio). En él se señala una corriente en dirección NE-SW. La parte superior de la capa (200-500 m) llarnada agua oriental es muy salina $(38,5 \% 0)$ y templada $\left(13,4^{\circ} \mathrm{C}\right)$ y la inferior, llamada agua septentrional, menos salada $(38,4 \% 0)$ pero más fría $\left(13^{\circ} \mathrm{C}\right)$. Posteriormente Funnestin \& Allain (1962) en otro estudio de la parte $\mathrm{NW}$ del mar Mediterráneo limitada al Sur por el paralelo $42^{\circ}$, o sea por encima de nuestra zona, señala que, la magnitud de tales capas varía considerablemente según la época del año $\mathrm{y}$, aún más, de unos años a otros. Así, en el otoño de 1958, cuando se hizo el estudio, la. 
capa oriental mostraba una gran magnitud a costa, al parecer, de la capa septentrional que aparecía como disminuida y cortada por la primera. La capa septentrional se supone que se forna en el golfo de León (TChernia, 1956 y Tchernia \& SAINT GUILy, 1959) por efecto de los vientos fríos y secos del Norte que a la rez concentran y enfrían el agua, aumentando su densidad y provoeando. por tanto, su bundimiento. Lógicamente este fenómeno debe ser muy acusado en los meses invernales, mientras que en el resto del año se produzca nuly débilmente.

Por otra parte se tiene la impresión de que, al menos en determinadas épocas del ano y posiblemente en los períodos de máxima producción biológica, hay una inversión del sentido de las corrientes medias y superficiales concebible en los siguientes términos: bundimiento de aguas superficiales al SE de la cuenca, flujo en profundidad en dirección E-W y afloramiento en esta posición para seguir después una direcciól próxima a $\mathrm{SW}-\mathrm{NE}$, como nosotros hemos podido comprobar en varios momentos de nuestro estudio (fig. 6), o sea una total inversión del flujo considerado tradicionalmente. Esta lipótesis puede compaginarse bien con las observaciones de Furnestix, si el flujo oriental llegase a ser cortado por la corriente septentrional precisamente en la época invernal.

Cuando llegan los meses invernales, muestra zona suele verse invadida, en mayor o menor escala según los años, por una masa de agua de origen profundo, ya que suele ser más densa que la que aparece durante el resto del año; sin embargo no se ha logrado aclarar el origen del agua invasora, ya que el punto de la toma de muestra se encuentra en el centro de la zona con evidente riesgo de mezcla con las aguas que fluyen durante el resto del año. Por otra parte, también existe la posibilidad de que el flujo de aguil septentrional vea obstaculizada su marcha por el flujo procedente del $\mathrm{SW}$, lo que daría lugar a un desbordamiento de las aguas profundas sobre la plataforma costera, si estos efectos se pudieran repetir varias reces en $\mathrm{nn}$ inismo ciclo anual, provocarían los diversos impulsos que suelen observarse generalmente $y$ según el tipo de agua que pledomine en el desbordamiento caracterizaria, y de becho caracteriza, a las distintas floraciones del ciclo. En el ciclo actualmente en estudio tenemos la sospecha de que el agua invasora. al menos en la prinera invasión, debía pertenecer al flujo septentrional, ya que las temperaturas eran inferiores en $0,5^{\circ}$ a las alcanzadas en cualquier época de ciclos anteriores, en valores absolutos de 12-12,50 durante el mes de febrero. Nuestras observaciones en el presente ciclo coinciden adeluás con la apreciación de una gran producción de agua septentrional en el golfo de León, durante este mismo invierno.

Sin embargo, tampoco debemos descartar que, cuando en un ciclo anual como el presente, en el que el flujo de las aguas pobre en sales nutrientes es desplazado por aguas profundas, el efecto del viento favo- 
rable al afloramiento ha de contribuir forzosamente a un aminto de la producción biológica.

Descripción del presente ciclo anual. Los meses de agosto a noviennbre transcurrierun en condiciones normales en relación con ciclos anteriores. En diciembre, tras la desaparición de la termoclina, aún permanecían elevadas las temperaturas, la salinidad comenzó a elevarse lasta las proximidades de $38 \%$, la concentración de fústuro pelnianecia baja y sopló viento favorable al afloramiento desde mediados de mes, que no se reflejic en las corrientes superficiales, ya que ćstas fluían de NH-SW.

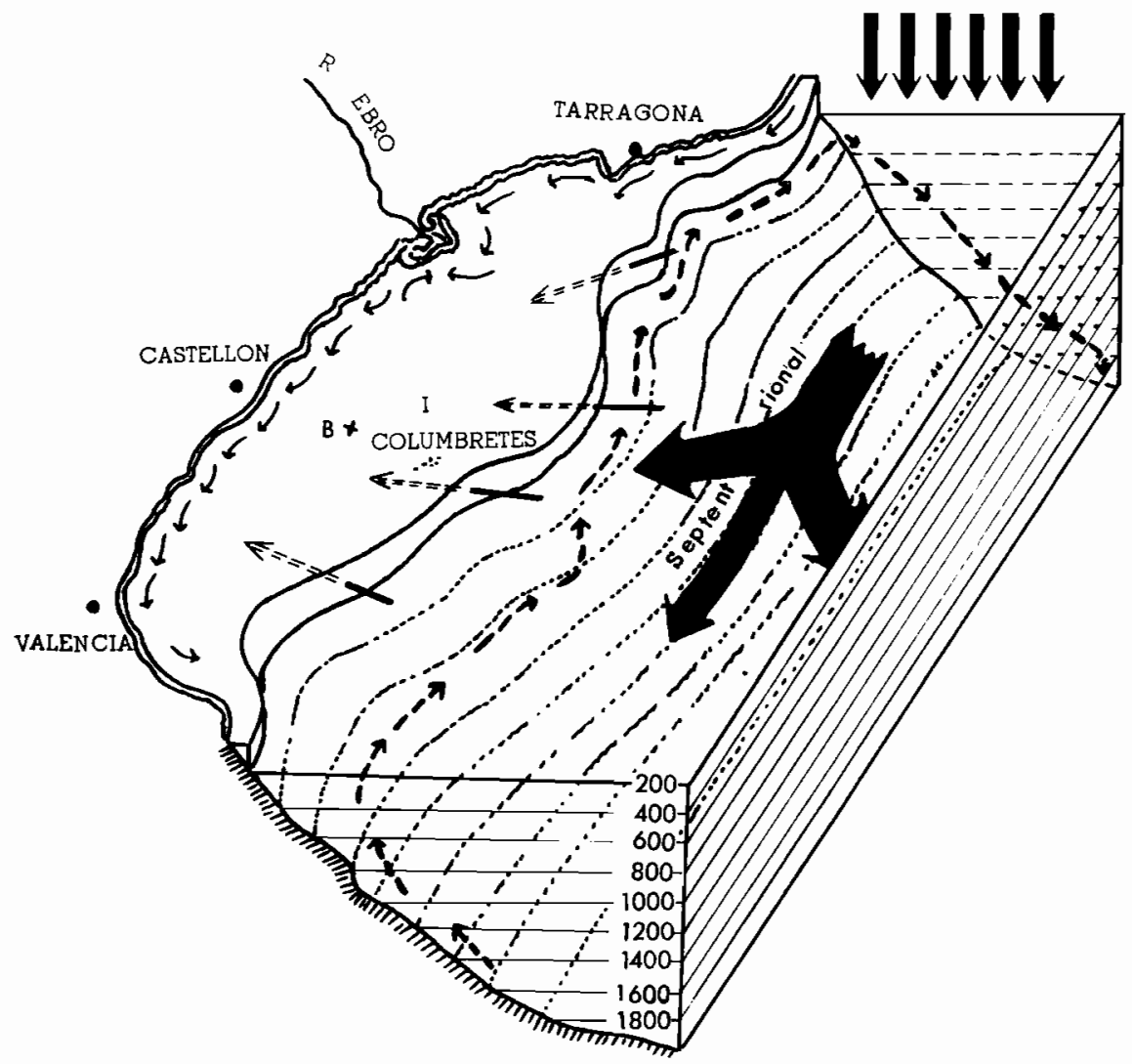

Frg. 6. - Mapa de la zona pescuera. Por debajo de la línea d: $200 \mathrm{~m}$ se representan las hipótesis sustentadas sobre dinámica de corrientes en el ciclo productivo. Las flechas gruesas, paralelas de la parte superior indican el descenso de aguas densas en el golfo de lueón, la flecha más gruesa y trifurcada muestra el posible curso de la corriente «septentrional» con sus inpulsos de aproximación a la costa, las invasiones se muestran por las flechas que se introducen en la capa de los $200 \mathrm{~m}$. Las flechas de trazos indican el curso de las corrientes ciclónicas remontadas al sur de la zona. 
En febrero, las temperaturas descienden hasta limites no conocidos en nuestra estación $\left(11,8-12,2^{\circ} \mathrm{C}\right)$, la salinidad se acerca más a $38 \%$ iniciándose una elevación de la concentración de fosfatos que aparece francamente notable a los $10 \mathrm{~m}$ superficiales. La transparencia del agua sigue disminuyendo y los vientos en los dias anteriores no predoininaron en las direcciones favorables al afloramiento. Como más adelante veremos, las aguas están extraordinariamente empobrecidas en fitoplancton. Estas características parecen indicar una primera invasión de la zona por aguas muy frias pero al mismo tiempo poco saladas. Sin embargo. es oportuno señalar que la temperatura del aire en el puerto durante este mes (MuÑoz, 196t) fue $1,4^{\circ} \mathrm{C}$ inferior a la media de los últimos años, pero no creemos que este efecto haya llegado a influir mucho sobre las bajas temperaturas registradas en el mar.

A finales de mes las aguas seguían frías, la salinidad aproximadamente igual, la transparencia seguía disminuyendo y en los días inmediatos comenzaron a soplar vientos favorables al afloramiento. También la concentración de fitoplancton permanecía baja.

En marzo, con temperaturas normales y relativamente uniformes. se aprecia una elevación de la salinidad hasta situarse sobre los $38,2 \%$, superior en el fondo que en la superficie. Las concentraciones de fósforo también se elevan considerablemente, más en el fondo y superficie que en las capas intermedias, seguramente porque en éstas pudo haber una asimilación intensa por parte del fitoplancton. La transparencia llega a los valores más bajos registrados por nosotros. Durante los quince días anteriores a la toma de inuestras soplaron vientos favorables al afloramiento y la corriente superficial era N.S. Parece que debe haberse producido una invasión de aguas profundas, probablemente favorecida por los vientos y cuyas características son : $\mathrm{S} 38,2 \% 0, t 13,0^{\circ} \mathrm{C}$, y $\sigma_{t} 28,85$ 28,95 , con notables cantidades de elementos nutrientes. ¿ Estamos en presencia de aguas septentrionales ayudadas a aflorar por el viento?

A principio de abril se observó un calentamiento del agua en los cincuenta metros superiores con salinidacles parecidas a las del mes anterior y desaparición de los fosfatos, rápidamente asimilados por el fitoplancton. Ja transparencia aumentó, prosiguiendo bajo los efectos de vientos favorables al afloramiento que provocaron corrientes en dirección E-W. A finales de mes las aguas estaban más calientes, la salinidad disminuyó en las capas superficiales y la concentración de fosfatos permaneció estacionaria o ligeramente más baja, siguió aumentando la transparencia y desde hacía varios días había cesado el flujo de vientos favorables al afloramiento. Estos hechos parecen indicar el predominio de las corrientes superficiales tradicionales, tratando de relegar a las aguas densas a capas más profundas.

A finales de majo se ruelve a formar la termoclina. A partir de los $5 \mathrm{~m}$ la salinidad ruelve a elevarse por encima de los $38 \%$, los fosfatos 
aumentan también, especialınente en el fondo y superficie y la transparencia vuelve a disminuir. Aunque en la segunda decena de mayo predominaron los vientos favorables al afloramiento su debilidad nos permite desestimar sus efectos. Lin nuevo impulso de la invasión de aguas de salinidad $38,2 \%$ parece producirse en las proximidades de la fecha en que se tomaron estas muestras, si bien la presencia de la termoclina, o la menor potencia del impulso, no debió permitir su afloramiento hasta la superficie. Esta rez la invasión del agua salada no fue ayudada por el viento. Aunque muy débilmente, también esta rez se acusaron estos efectos sobre la producción biológica. I primeros de julio persisten los efectos observados un mes antes aunque más debilitados, aún se acusan salinidades de $38 \%$ a partir de $\operatorname{los} 20 \mathrm{~m}$ y $0,09 \mu \mathrm{g}$ atom. $\mathrm{P} / \mathrm{l}$ en $75 \mathrm{~m}$. A finales de mes apenas son perceptibles estos efectos.

\section{FITOPLANCTON}

Para su determinación se han seguido las mismas técnicas que en años anteriores: filtración de unos $11 \mathrm{l}$ de agua de mar, extracción del filtrado con acetona al $90 \%$ en caliente y medida de la densidad óptica del extracto. A partir de los datos primarios espectrofotométricos, adecuadamente corregidos cuando fuese necesario, se obturieron los correspondientes valores de concentración de pigmentos mediante la aplicación de las fórmulas de Richards \& Thompson (1952). En la tabla publicada como apéndice se dan los valores numéricos de las concentraciones de pigmentos, también se incluye, aparte de los datos oceanográficos e hidrográficos correspondientes, los valores corregidos de las observaciones

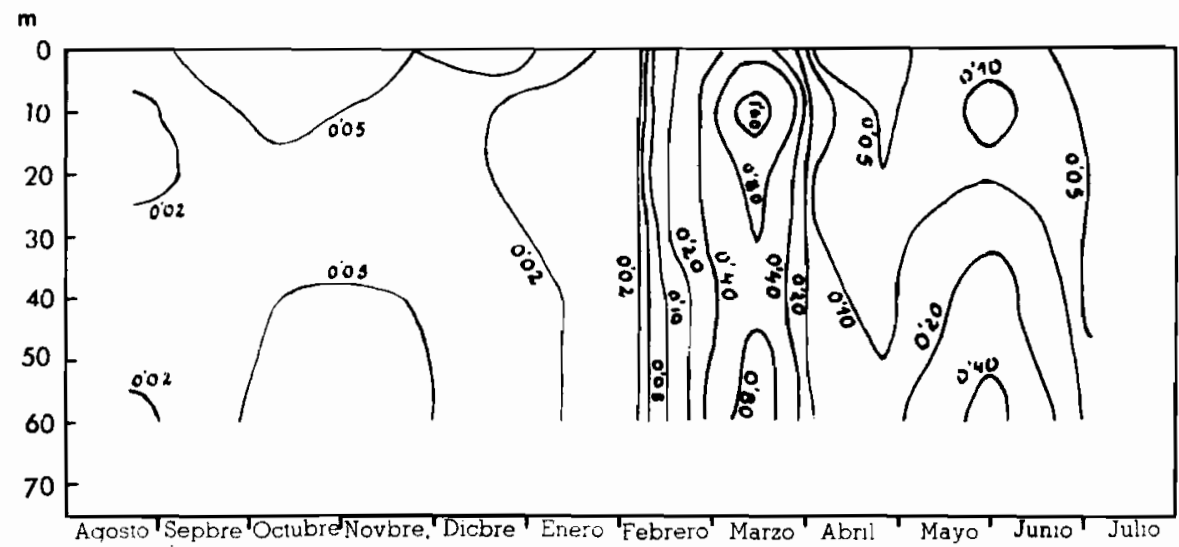

FIg. 7. - Concentración de clorofila a en $\mathrm{mg}_{/}^{\prime} \mathrm{m}^{3}$ en la estación $\mathrm{B}$, calculada segín la fórmula de Richards y Thompson, de ngosto de 1962 a julio de 1963 . A la izquierda, escala de profundidades en metros. 
espectrofotométricas a 430 y $655 \mathrm{~m} 1 \mathrm{\mu} \mu$, asi como sus cocientes y el valor calculado en unidades patrón Harvey de cada determinación.

En la figura 7 se representa la concentración de clorofila $a$ durante el presente ciclo anual. Como puede observarse, ésta permanece baja durante los ineses estivales y otoñales, sin embargo lo más notable es la casi desaparición en la determinación del 6 de febrero, para quince días más tarde producirse un apreciable aumento que culmina en la determinación del 15 de marzo con los máximos valores registrados hasta la fecha, especiahuente el ralor $1,99 \mathrm{mg}$ a $10 \mathrm{~m}$ que es el valor nás alto encontlado desde que se comenzó este tipo de estudios a finales de 1957. En el mes siguiente lus valores vuelien a ser bajos, notándose un apreciable aumento en el mes de mayo.
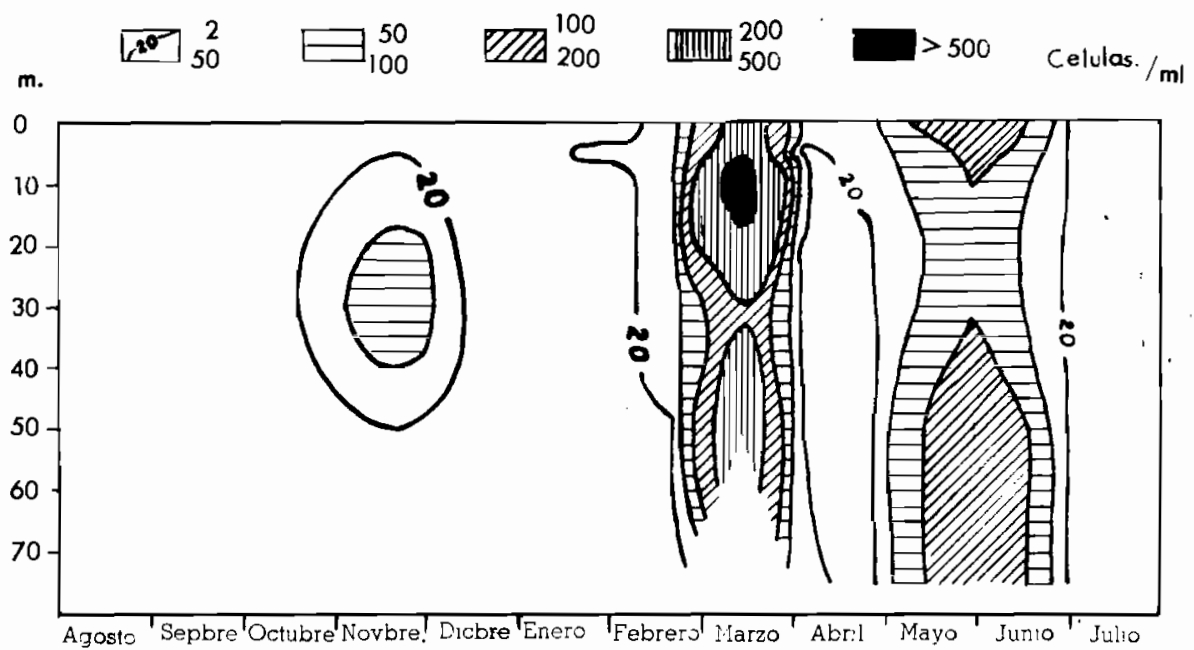

FIG. 8. - Concentración total de fitoplancton, en células por ml, contadas al mi(roscopio de Ttermöh], dirante el periodo de estudio. A la izquierda, escala de profundidades on metros.

Ofrecemos timubién en la figura 8 la distribución por niveles, durante el período de estudio, de la concentración celular expresada en número de células por mil, contadas al microscopio de Utermöhl. El recuento la sido realizado jor el $D_{1}$. R. Margalef $y$ es una contribución que arreciamos en todo $\mathrm{su}$ ralor, asi como sus consejos y la lectura del manuscrito, por todo lo cual le hacemos patente, una vez más, nuestro profundo agradecimiento. El estudio descriptivo del fitoplancton de red lo incluirá el autor en otra comunicación.

La figura I se lia construido con los valores obtenidos del cociente $\mathrm{D}_{430} / \mathrm{D}_{\mathbf{6} 65}$ y da una idea el el tiempo $y$ en el espacio del envejecimiento de las poblaciones durante el invierno, preferentemente en aguas de pro- 
m.

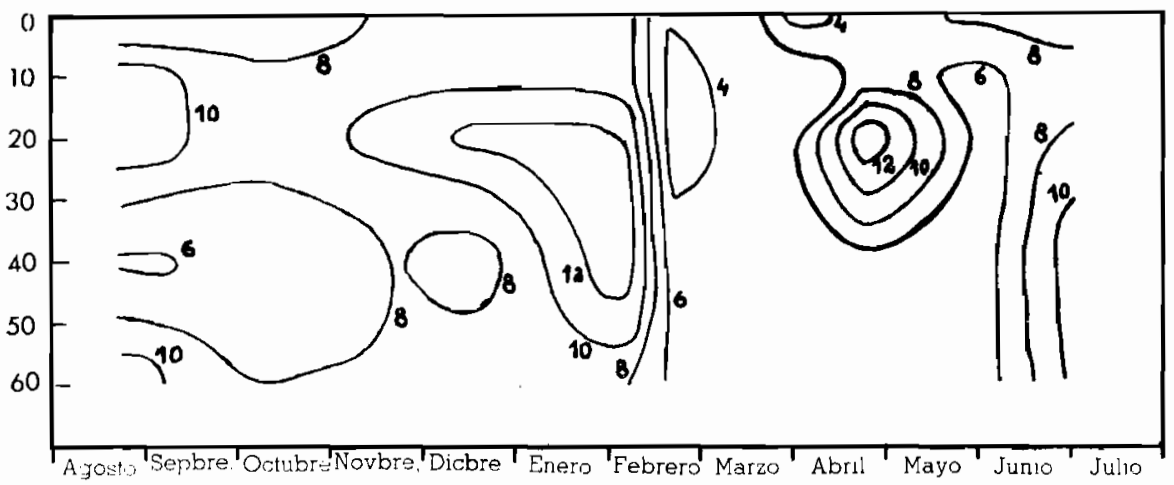

Fig. A. - Distribución de los valores del cociente $\mathrm{D}_{430} / \mathrm{D}_{\mathrm{bi}}$ en la estación $\mathrm{B}$, de agosto de 1962 a julio de 1963. A la izquierda, escala de profundidades en metros. Los valores inferiores representan estados más juveniles $\mathrm{v}$ con mayor productividad. Por encima del valor $i$ os muy probable la presencia de carotinoides de origen animal on las muestras.

fundidad media, así como su renovación y rejuveneciniento durante la primavera. También a través de dicho cociente se señala en la figura 10 el curso de la sucesión, en este caso cada punto representa el valor medio de los cocientes obtenidos a distintas profundidades. Fácilmente se observa que en la segunda determinación de febrero aparece un vivo rejuvenecimiento de la población, seguido de un aumento notable de la biomasa en la determinación siguiente del mes de marzo. En esta figura los bajos valores superficiales de abril y mayo se compensan con los aún altos del fondo, con lo que el tono general del segundo máximo pasa casi inadvertido en la figura.

En un trabajo anterior de psta serie (NARGalef \& HERRERA. 1963) se calculó la productividad para aquel ciclo anual, la cual parece resultó inferior a las suposiciones previstas. Alora, teniendo en cuenta la importancia que para nuestra zona pesquera representa el cálculo de dicha producción, presentamos unos valores de la productividad en $\mathrm{g} / \mathrm{m}^{2}$ que estimamos bastante aproxinados (tabla IV).

Wl cálculo resultia de la expresión: $\Sigma_{i: 3}^{\circ} 403 \cdot m \cdot D_{\text {firs }}$ entre cero y 7.) 11 , límite de profundidad estimada para la estación oceanográfica, en la que $m$ representa la altura en metros a que corresponde la densidad óptica observada a $665 \mathrm{~m} \mu$ : $\mathrm{D}_{6,5,5}$ representa el valor de la densidad óptica a esa longitud de onda de un extracto cetónico que en $1 \mathrm{ml}$ contiene los pigmentos de $1 \mathrm{l}$ de agua filtrada : 403 es un coeficiente obtenido combinando el valor 500. propnesto por XIRGALEF (1960), que posteriormente estimó exagerado y el fue resultaría. al aplicar una fórmula tan 


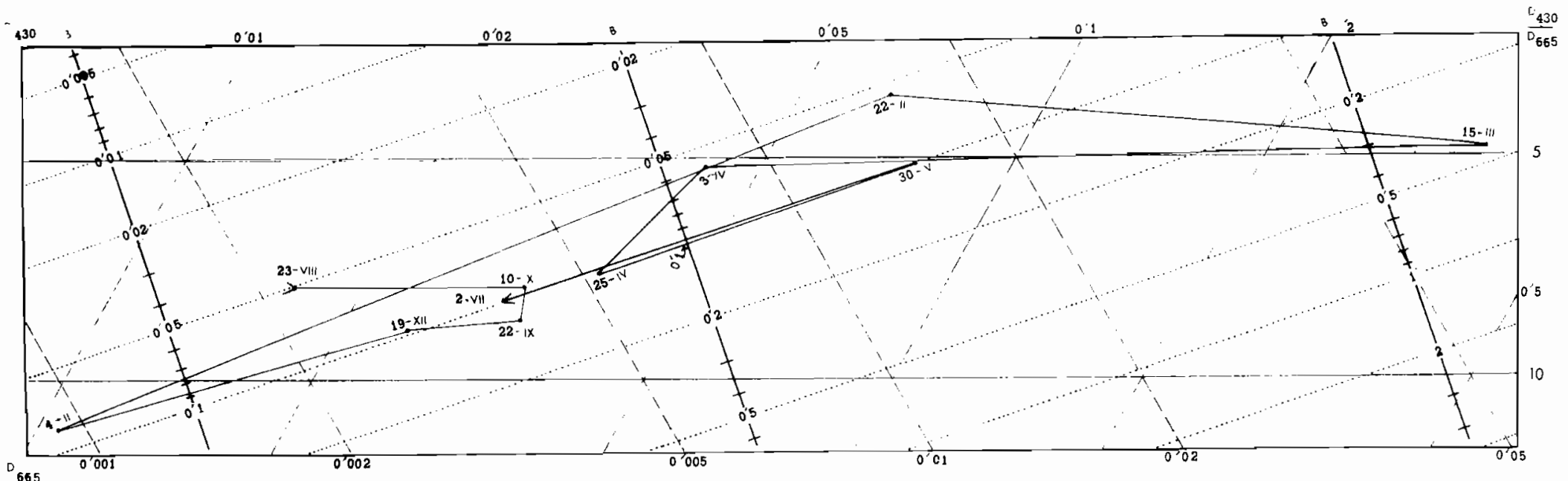

Fig. 10. - Tos scgmentos rectos unen puntos que corresponden a fechas consecutivas. Estos puntos vienen definidos por los valores medios de las observaciones espectrofotométricas a 431 , 665 m m (referidas a extractos cetónicos que en 1 ml contienen los pigmentos de 1 l de agua) a distintas profundidades en la estación B. Para otras explicaciones en relación con esta figura ver Margalef (1960). 
T A B I.A I V

\begin{tabular}{|c|c|c|}
\hline lichi & 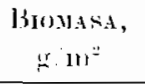 & 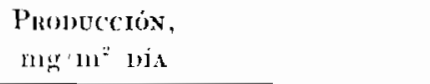 \\
\hline $23-V I I I-62$ & $1,(6 i)$ & 61,7 \\
\hline $10-X-1 ; 2$ & 3,69 & 114,9 \\
\hline $2 \cdot 2-X T-62$ & 3,68 & 114,5 \\
\hline 19-XIT-62 & 2,34 & 72,7 \\
\hline 6-II-63 & 0.97 & 30,1 \\
\hline 22-II-63 & 5.69 & $+332,6$ \\
\hline $17-1[1-63$ & 37.76 & 2156,6 \\
\hline $3-I V-63$ & 5,41 & 243,3 \\
\hline 25-IV-(0;3 & 5,01 & 173.2 \\
\hline $30-V-63$ & 9,56 & 539.6 \\
\hline 2 -VII-633 & 2.93 & $119.1)$ \\
\hline Modia anual & $\overline{7}, 14$ & $372,4 \mathrm{mg} / \mathrm{m}^{2} \in \mathrm{n}$ p'so seco \\
\hline Media auual (n carbono & 3,11 & $59,1 \mathrm{~g} / \mathrm{m}^{2}$ año en carbono \\
\hline
\end{tabular}

simple como la de Margalef ( $\mathrm{P}=5 \% 0 \mathrm{D}_{665}$ ), al valor obtenido aplicando la fórmula de Rither \& Y.ivrsch (1957) a los mismos datos :

Producción media ciclo 1959-1960, ng $/ \mathrm{m}^{2}$ día según R y Y, 110.

Producción media ciclo 1959-1960, mg/m² día según M, 180.

$$
\frac{500 \cdot 110}{1 \times(1)}=30.5,5 \quad f=\frac{500+305,5}{2} \simeq 403
$$

Hemos de aclarar", por otra parte, que este coeficiente no tiene otra significación que el de seguir un procediniento análogo al empleado con los datos del ciclo 1959-1960, para poder establecer comparaciones. La producción media del presente ciclo viene a ser unas dos y media veces superior a la del ciclo 1959-1960 y la mitad de la aportación corresponde al mes de marzo. No hemos tenido en cuenta más que once meses, pero el que falta, septiembre, aunque habría rebajado algo el valor medio mensual, el resultado final no hubiera sido muy diferente.

En la misma tabla se dan los valores de la biomasa en $\mathrm{g} / \mathrm{m}^{2}$ obtenidos también por integración de los valores correspondientes a los distintos niveles y aplicando las fórmulas dadas por MARGALFF (1960) en el citado trabajo. Aquí, la producción es tres veces superiol a la del ciclo anual 1959-1960 y el elevado valor alcanzado es consecuencia. comno en lia producción media, de la determinación de marzo.

Aplicación de nuevas fórmulas al cálculo de la concentración de pigmentos en el fitoplancton. Trabajos experimentales en diferentes laboratorios han mostrado ciertas discrepancias con los resultados obtenidos al aplicar las fórmulas de Richards y Thompson para el cílculo de los 
pigmentos en el fitoplancton. Así, los coeficientes dados para el cálculo de las clorofilas $a$ y $b$ en las citadas fórmulas son bajos, con lo que los valores resultantes aparecen demasiado elevados. Ta clornfila $c$. la más abundante, no viene en la citada fórmula en unidades cegesimales, sino en otras convencionales y estrictantente no comparables con la empleada en las restantes clorofilas. Igual puede decirse de los carotinoides. los cuales debido a que la unidad de comparación es realmente superior al gramo al equipararlos a esta unidad resulta menospreciada la cantidad real de carotinoide. Finalmente los pigmentos del grupo de la xantofila: piridina y fucoxantina contribuyen a incrementar el total de carotinoides de origen animal si se aplican las fórmulas de Richatds y Thompson, a pesar de ser componentes del fitoplancton.

Para intentar superar estas deficiencias se han emprendido trabajos en varios laboratorios y últimamente PARson \& STrickind (1963), después de un estudio experimental, han presentado nuevas fómnulas para el cálculo de los pigmentos clorofílicos. También indican que si se hace pasar el agua de filtración, previamente, a través de ma malla grosera que retenga el zooplaucton, el extracto obtenido después de la filtración contendrá solamente los pigmentos de origen vegetal, incluidos la. piridina y la fucoxantina, con lo cual mediante una sencilla fórmula se. puede obtener un valor más real de los carotinoides de origen vegetal.

Nosotros hemos querido ohservar las diferencias existentes en lós. valores numéricos al emplear ambas fórumlas. Se observa, en primer lugar que, il iplicar las fórmulas de Richards y Thompson aparecen menos valores negativos. No existe relación constante para un mismo extracto calculado por ambas fómulas y las diferencias aparecen intimamente ligadas con el estado de la población; las diferencias son inínimas cuando la concentración de pigmentos es máxina (tabla V), apareciendo muy próximos en este estado las cantidades de clorofila $\boldsymbol{b}$ calculadas por ambos métodos: lá cantidad de clorofila a siempre resulta menor aplicando las fórmulas de Parsons y Strickland y el factor de conversión oscila entre 1,33 y 1,42 ; igual ocurre con la clorofila c que para su conversión de gramos a unidades convencionales es preciso multiplicar por un factor 1,95 a 2,5. En los demás estados de las poblaciones los factores de conversión son mucho mayores, llegando a alcanzar valores de 4,5 para la clorofila $a, 2,7$ para la $b$ y 4 para la $c$, desestimando algunos a todas luces anormales.

En la tabla $V$ se incluyen también los carotinoides de origen regetal calculados según las fórmulas de Parsons y Strickland, si bien no deben ser tenidos muy en cuenta estos valores, puesto que no fueron separados los carotinoides de origen animal, según prescriben los autores para poder hacer uso de sus fórmulas.

En consideración al hecho de que al aplicar las fórmulas de Parons y Strickland los vilores obtenidos para todas las clorofilas vienen expre- 
T A B L A V

Concentraciones de lis distinta clemefilas calculadas segúu las formulas de Richardiz \& Thompson (1952) y Parsons \& Strickland (1963). Cociente de los valores calculados por ambos métodos. Concentración de carotinoides calculada según la fórmula de Parson: y Strickland y relación de los distintos pigmentos a la clorofila a calculados scgún esta última fórmula.

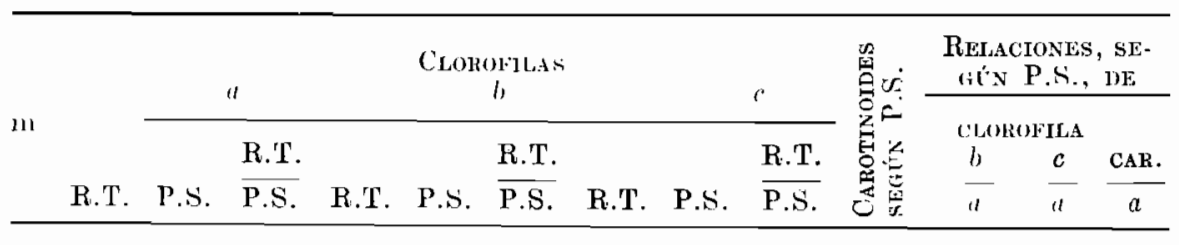

$2: 3-1$ III- 62

$\begin{array}{rlllllllllll}0 & 0,04 & 0,01 & 4,09 & 0,05 & 0,03 & 1,67 & 0,30 & 0,08 & 3,90 & 3,24 & 8,36 \\ 10 & 0,01 & & & 0,01 & 0,01 & 2,14 & 0,30 & 0,10 & 2,92 & & \\ 20 & 0,01 & & & 0,02 & 0,01 & 2,21 & 0,20 & 0,06 & 3,47 & & \\ 40 & 0,05 & 0,02 & 2,06 & & & & 0,22 & 0,10 & 2,94 & & 4,08 \\ 60 & 0,01 & & & & & & 0,49 & 0,20 & 2,52 & & \end{array}$

$10-\mathrm{X}-62$

$0 \quad 0,07$

$0.03 \quad 2,12$

$200 \quad 0,04 \quad 0,01 \quad 2,44$

$40 \quad 0,05 \quad 0,02 \quad 2,08$

$60 \quad 0,06 \quad 0,03 \quad \mathbf{1 , 8 7}$

$0,07 \quad 0,06 \quad 1,25$

$0,03 \quad 0,03 \quad 1,12$

$\begin{array}{lll}0,45 & 0,15 & 2,98\end{array}$

$0,49 \quad 0,19 \quad 2,54$

$\begin{array}{lll}0,28 & 0,10 & 2,98\end{array}$

$0,02 \quad 0,01 \quad 1,86$

$0,01 \quad 0,00 \quad 2,1.1$

0,26

$0,09 \quad 3,10$

$0,35 \quad 0,13 \quad 2,76$

0,09

0,13

0,03

0,05

$1,18 \quad 4,81 \quad 2,73$

$0,97 \quad 6,11 \quad 4,21$

$6,49 \quad 2,33$

$0,48 \quad 3,73 \quad 2,20$

$22-\mathrm{XI}-62$

$0 \quad 0,05$

$40 \quad 0,05$

$0,02 \quad 2,52$

$0,04 \quad 0,02 \quad 1,79$

$0,03 \quad 0,02 \quad 1,97$

0,2

$\begin{array}{lll}0,06 & 3,98\end{array}$

$0,20 \quad 0,05 \quad 4,08$

$0,18 \quad 0,05 \quad 3,38$

0,13

0,09

$1,18 \quad 3,36 \quad 6,90$

$\begin{array}{lllllll}60 & 0,06 & 0,03 & 1,77 & 0,03 & 0,02 & 1,64\end{array}$

19-XII-62

$\begin{array}{llll}0 & 0,07 & 0,03 & 1,89\end{array}$

$10 \quad 0,02$

$20 \quad 0,02$

$40 \quad 0,04 \quad 0,02 \quad 2,52$

$60 \quad 0,03 \quad 0,01 \quad 4,09$

$0,04 \quad 0,03 \quad 1,56^{3}$

$0,27 \quad 0,08 \quad 3,35$

$0,32 \quad 0,10 \quad 3,06$

$0,03 \quad 0,02 \quad 1,57$

$0,05 \quad 0,03 \quad 1,58$

$\begin{array}{lll}0,03 & 0,02 & 1,79\end{array}$

$0,27 \quad 0,07 \quad 3,83$

$0,22 \quad 0,05 \quad 3,98$

$0,03 \quad 0,02 \quad 1.67$

$0,21 \quad 0,05 \quad 3,90$

$0,06 \quad 0,75 \quad 2,32 \quad 1,66$

0,02

$0,00 \quad 1,18 \quad 3,36 \quad 0,00$

$3,24 \quad 8,36$

\section{6-II-63}

$$
\begin{array}{ll}
0 & \\
10 & 0,01 \\
20 & 0,01 \\
40 & 0,00 \\
60 & 0,01
\end{array}
$$

$\begin{array}{lll}0,12 & 0,01 & 13,29\end{array}$

$0,13 \quad 0,022 \quad 8,36$

$\begin{array}{lll}0,26 & 0,08 & 3,43\end{array}$

\begin{tabular}{|c|c|c|c|c|c|c|c|c|c|c|c|c|}
\hline 0,25 & 0,17 & 1,42 & 0,09 & 0,08 & 1,14 & 0,53 & 0,22 & 2,37 & 0,27 & 0,45 & 1.23 & 1,53 \\
\hline 0,29 & 0,20 & 1,42 & 0,12 & 0,11 & 1,05 & 0,86 & 0,38 & 2,29 & 0,10 & 0,56 & 1,85 & 0,49 \\
\hline 0.17 & 0,12 & 1,46 & 0,05 & 0,05 & 1,17 & 0,42 & 0,17 & 2,46 & $(0,19$ & 0,40 & 1,48 & 1,66 \\
\hline 0.15 & 0,10 & 1,48 & 0.09 & 0.03 & 1,21 & 0.40 & 0,16 & 2.49 & 11.20 & 0.25 & 1,60 & 1,97 \\
\hline
\end{tabular}

$\begin{array}{lll}0,30 & 0,08 & 3,76\end{array}$

$0,22 \quad 0,06 \quad 3,48$

22-II-63 
TABLA Y (Continuación.)

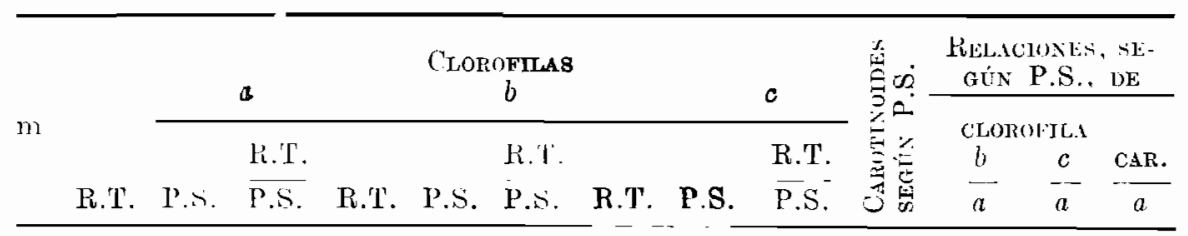

15-1II- 6 ;

$\begin{array}{rlllllllllllll}0 & 0,56 & 0,42 & 1,35 & 11,30 & 0,30 & 1,00 & 1,59 & 0,76 & 2,10 & 0,58 & 0,71 & 1,82 & 1,87 \\ 10 & 1,99 & 1,49 & 1,33 & 1,51 & 0,53 & 0,95 & 5,00 & 1,59 & 1,96 & 2,89 & 0.36 & 1,02 & 1,93 \\ 20 & 0,97 & 0,73 & 1,34 & 0,2 \times & 0,28 & 1,00 & 1,52 & 0,75 & 2,01 & 1,20 & 0,39 & 1,04 & 1,66 \\ 40 & 0,68 & 0,50 & 1,37 & 0,30 & 0,25 & 1,20 & 0,44 & 0,18 & 2,42 & 0,78 & 0,51 & 0,37 & 1,57 \\ 60 & 1,14 & 0,85 & 1,35 & 0,54 & 0,44 & 1,21 & 0,19 & 0,08 & 2.39 & 1,37 & 0,52 & 0,10 & 1,61\end{array}$

3-IV-6.3

\begin{tabular}{|c|c|c|c|c|c|c|c|c|c|c|c|}
\hline 0,04 & 0,02 & 2,54 & 0,05 & & & & & $0,0 t$ & & & 3,25 \\
\hline 0.13 & 0,05 & 2,92 & 0,06 & & 0,31 & & & 0,24 & & & \\
\hline 0,08 & 0,04 & 1,79 & 0,03 & $0,01 \quad 2,60$ & 0,10 & & & 0,14 & $0,2 \mathrm{~S}$ & & \\
\hline 0,13 & 0,08 & 1,60 & 0,02 & $0,00 \quad 11,69$ & 0,08 & & & 0,29 & 0,03 & & \\
\hline 0.20 & 0,14 & 1,47 & 0,11 & $0,09 \quad 1,23$ & 0,43 & $0.16 j$ & 2,68 & 0,18 & 0,63 & 1.16 & \\
\hline
\end{tabular}

25-IV-63

$\begin{array}{rlllllllllllll}0 & 0,04 & 0,02 & 2,57 & 0,05 & 0,04 & 1,44 & 0,32 & 0,10 & 3,29 & & 2,13 & 5,67 & \\ 10 & 0,04 & 0,01 & 3,78 & 0,03 & 0,01 & 2.69 & 0,20 & 0,03 & \mathbf{5 . 9 7} & & 1,18 & 3.36 & \\ 20 & 0,05 & 0,02 & 2,11 & 0,06 & 0,03 & 2,18 & 0,35 & 0,12 & 2,98 & 0,29 & 1,08 & 4,815 & 11,72 \\ 40 & 0,07 & 0,04 & 1,66 & 0,05 & 0,04 & 1.04 & 0,53 & 0,22 & 2,42 & 0,00 & 1,04 & 5,19 & 0,00 \\ 60 & 0,13 & 0,08 & 1,57 & 0,08 & 0,07 & 1.17 & 0,32 & 0,11 & 3,08 & 0,14 & 0.89 & 1,26 & 1,69\end{array}$

30. V. -63

$\begin{array}{rrrlllllllllll}0 & 0,08 & 0,04 & 1,85 & 0,02 & 0,01 & 2,20 & 0,36 & 0,12 & 3,12 & 0,15 & 0,25 & 2,61 & 3,44 \\ 10 & 0,12 & 0,08 & 1,59 & 0,05 & 0,04 & 1,32 & 0,39 & 0,14 & 2,80 & 0,10 & 0,55 & 1,83 & 1,29 \\ 20 & 0,09 & 0,05 & 1.77 & 0,04 & 0,03 & 1,65 & 0,30 & 0,09 & 3,38 & 0,018 & 0,51 & 1,73 & 1,45 \\ 40 & 0,27 & 0,19 & 1.46 & 0,06 & 0,05 & 1,27 & 0,53 & 0,21 & 2,53 & 0,36 & 0,25 & 1,12 & 1,94 \\ 60 & 0,48 & 0,35 & 1,38 & 0,12 & 0,12 & 1,04 & 0.80 & 0,40 & 2,17 & 0,57 & 0,34 & 1,15 & 1,65\end{array}$

2.VII-63

$\begin{array}{rrrrrrrrrrrrrr}0 & 0,03 & 0,01 & 4,50 & 0,05 & 0,04 & 1,30 & 0,46 & 0,20 & 2,29 & 0,07 & 5,64 & 29,04 & 9,85 \\ 10 & 0,04 & 0,02 & 2,52 & 0,03 & 0,02 & 1,79 & 0,22 & 0,05 & 3,98 & & 1,18 & 3,36 & 3,45 \\ 20 & 0,05 & 0,03 & 2,08 & 0,06 & 0,06 & 1,06 & 0,68 & 0,28 & 2,48 & & 2,18 & 10,62 & \\ 40 & 0,04 & 0.01 & 3.76 & 0,02 & 0,01 & 2.90 & 0.30 & 0.09 & 3.51 & 0,10 & 0,75 & 0,89 & 10,00 \\ 60 & 0,07 & 0,04 & 1,89 & 0,06 & 0,04 & 1,04 & 0,37 & 0.12 & 2,99 & 0,14 & 1,20 & 3,36 & 3,94\end{array}$

Nota: Ios cocientes se han obtenido a pautir di los valul's sin redondear.

sados en las mismas unidades, hemos empleado estos valores para el cálculo de lo cocientes de los restantes pigmentos a la clorofila $a$. Tuos valores resultantes se incluyen en la citada tabla. Las figuras 11,12 y 13 muestran gráficanente la distrilunción de estos cocientes en el espacio $y$ en el tiempo. 
m.

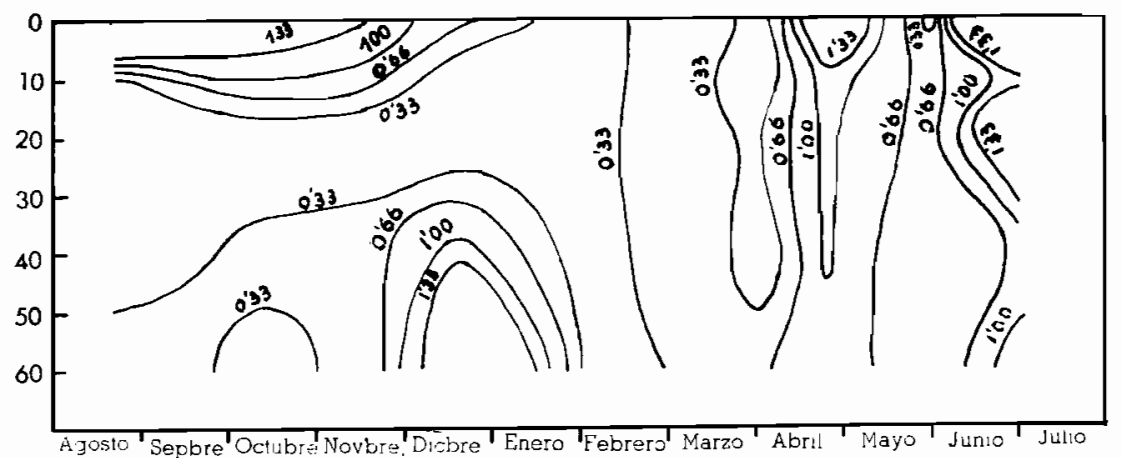

Fig. 11. - Concentración de clorofila $b$ relativa a la clorofila a ( $\mathrm{mg} / \mathrm{mg}$ ) en la estación B, de agosto de 1962 a julio do 1963 , calculadas según las fórmulas de Parsons y Strickland. A la izquicrda, escala de profundidades en metros.

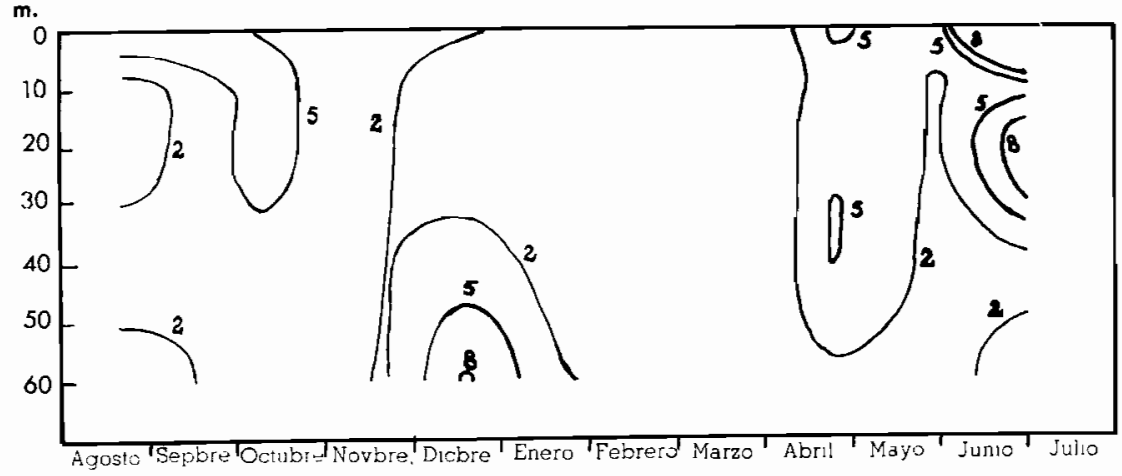

Fig. 12. - Concentración de clorofila $c$ relativa a la clorofila a ( $\mathrm{mg} / \mathrm{mg}$ ) en la estación B, de agosto de 1962 a julio de 1 !k33, calculadas según las fórmulas de Parsons y Strickland. A la izquierda, escala de profundidades en metros.

m.

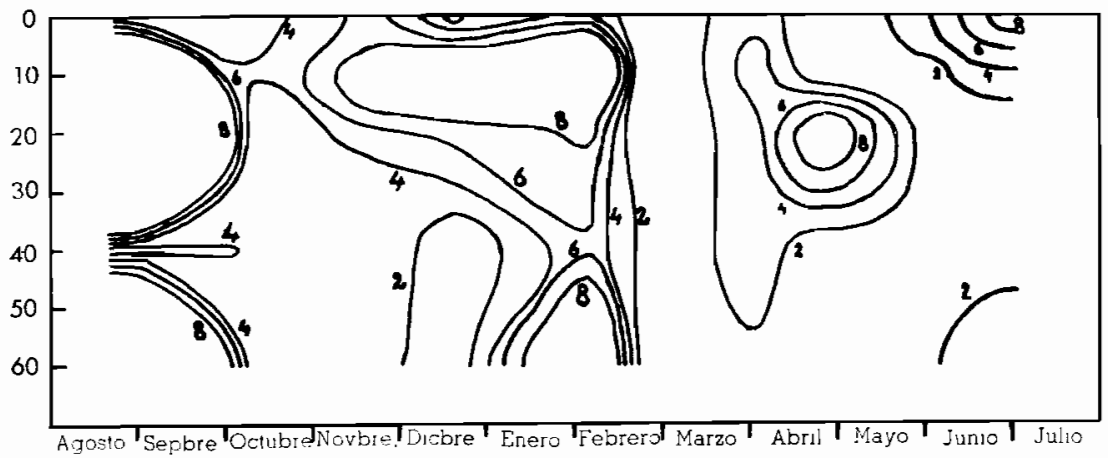

Fia. 13. - Concentración de carotinoides relativa a la clorofia a (unidades/mg) en la estación $B$, de agosto de 1962 a julio de 1963 , calculadas según las fórmulas de Parsons $y$ Strickland. A Ia izquicria. escala de profundidades en metros. 
También hemos aprovechado la homogeneidad de las unidades de medida de los distintos pigmentos para considerar sus variaciones a lo largo del año. Los valores numéricos se dan en la tabla VI y de ellos se saca en conclusión que, a través de toda la columna de agua la clorofila a varía de 0,000 a $0.798 \mathrm{mg} / \mathrm{u}^{3}$ lo que refleja una característica

T A B L A V I

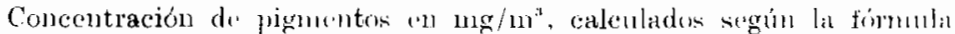
de Parsous \& strickland (1963)

\begin{tabular}{|c|c|c|c|c|c|}
\hline & & \multicolumn{3}{|c|}{ CLOROFILAS } & \multirow{2}{*}{$\begin{array}{l}\text { ('AROTI- } \\
\text { NOIDES }\end{array}$} \\
\hline & & $a$ & $b$ & $c$ & \\
\hline $\begin{array}{l}\text { Valor medio a lo } \\
\text { largo de toda la } \\
\text { columna }\end{array}$ & $\begin{array}{c}93-\mathrm{VIII} \\
10-\mathrm{X} \\
2 \cdot 2-\mathrm{XI} \\
19-\mathrm{XII} \\
6-\mathrm{II} \\
22-\mathrm{II} \\
15-\mathrm{III} \\
3-\mathrm{IV} \\
25-\mathrm{IV} \\
30-\mathrm{V} \\
2-\mathrm{VII}\end{array}$ & $\begin{array}{l}0,006 \\
0,024 \\
0,027 \\
0,012 \\
0,000 \\
0,147 \\
0,798 \\
0,066 \\
0,034 \\
0,142 \\
0,022\end{array}$ & $\begin{array}{l}0,010 \\
0,020 \\
0,020 \\
0,024 \\
0,026 \\
0,067 \\
0,360 \\
0,020 \\
0,038 \\
0,050 \\
0,034\end{array}$ & $\begin{array}{l}0,108 \\
0,132 \\
0,053 \\
0,070 \\
0,050 \\
0,232 \\
0,640) \\
0,032 \\
0,114 \\
0,192 \\
0,148\end{array}$ & $\begin{array}{l}0,000 \\
0,076 \\
0,106 \\
0,016 \\
0,0141 \\
0,190 \\
1,404 \\
0,182 \\
0,080 ; \\
0,250 \\
0,062\end{array}$ \\
\hline $\begin{array}{l}\text { Valor medio a In } \\
\text { largo de todo el } \\
\text { вño, por niveles }\end{array}$ & $\begin{array}{cc}0 \mathrm{~m} \\
10 & \gg \\
20 & \gg \\
40 & \gg \\
00 & \gg\end{array}$ & $\begin{array}{l}0,070 \\
0.153 \\
0,107 \\
0,094 \\
0,148\end{array}$ & $\begin{array}{l}0,050 \\
0,077 \\
0,057 \\
0,045 \\
0,079\end{array}$ & $\begin{array}{l}0.162 \\
0,240 \\
0,203 \\
0,113 \\
0,130\end{array}$ & $\begin{array}{l}0,153 \\
0,373 \\
0,186 \\
0,169 \\
0,235\end{array}$ \\
\hline
\end{tabular}

del fitoplancton del grupo de las diatomeas, no solamente por su amplia variación de épocas de máxima a mínina productividad, sino por reducirse a cantidades inapreciables en estas últimas: la clorofila $b$ de 0,010 a $0,360 \mathrm{mg} / \mathrm{m}^{3}$ apareciendo como característica del grupo de las dinoflageladas, siempre existentes en el mar y menos expuestas a fuertes explosiones en las épocas de máxima producción : la clorofila $c$ de 0,320 a $0,660 \mathrm{mg} / \mathrm{m}^{3}$ aparece como una car'acteristica más típica aún de las dinoflageladas, ya que solamente duplica sus valores; finalmente. los carotinoides del fitoplancton. que rarían de 0,000 a $1,404 \mathrm{mg} / \mathrm{m}^{3}$, al igual que la clorofila $a$, aparece como un pigmento característico de las diatomeas. A los distintos nireles considerados a lo largo del año, el de $10 \mathrm{~m}$ presenta la máxima concentración de pigmentos, después disminuye en los siguientes 20 y $40 \mathrm{~m}$, para volver a aumentar a los $60 \mathrm{~m}$ hasta casi alcanzar el primer máximo para cada uno de los pigmentos individualmente considerados, sin embargo la clorofila $b$ le sobrepasa, esto está de acuerdo con su característica resistencia a la descomposición, acompalñando en su mayor parte al plancton muerto cuando se sedimenta. 
Unidades patrón Harvey en relación con la clorofila y los pigmentos en general. En uno de los trabajos anteriores de esta serie (MARGalef, Herrera \& ARIas, 1959) se establecieron las relaciones entre la longitud de onda a $430 \mathrm{~m} \mu$ y la clorofila $a$ con el número de unidades patrón Harvey (UPH) oltenidas por comparación visual con patrones coloreados inorgánicos, limitándose sílo a esto porque los restantes pigunentos, excepto la clorofila $b$, no renían expresados en unidades cegesimales. l'nesto que esta dificultad es parciahnente superada aplicando las fórmulas de Parsons ! Strickland, hemos calculado las relaciones clorofila a /UPH, clorofila total / $\mathrm{TPH}$, pigmentos totales /UPH ` clorofila $a$, según las fórmulas de Richards y Thompson / UPH. Los valores en LPH fueron calculados mediante la fómula establecida en el trabajo citado anteriormente : $\left[\mathrm{PH}=35,7 \mathrm{D}_{430}\right.$, ya que 110 disponemos de determinaciones colorimétricas directas.

La última relación presenta una ecuación : UPH $=11620 \mathrm{H1g}$ clorofila $a+380$, algo diferente de la que se publicó en 1959 (LPH $=$ $=7500 \mathrm{mg}$ clorofila $a+500)$ con lo que se pone de manifiesto que todos los valores medios que se obtengan manejando este tipo de datos numéricos está intimanente relacionado con la sucesión histórica de las poblaciones de fitoplancton. Las senaladas discrepancias nos sugieren que, no se debe exagerar su significación en las hipótesis de trabajo. basadas en las determinaciones colorimétricas y expresadas en UPH, de estudios anteriores.

Ia figura 14 nos muestra la relación entre la cantidad de clorofila a contenida en $1 \mathrm{~mol}$ de extracto por I de agua filtrada y las correspondientes UPH. I a recta viene expresada por la ecuación : UPH $=15145 \mathrm{mg}$ clorofila $a+685$, o sea que $1 \mathrm{~g}$ de clorofila $a$ puede equivaler, según esta ecuación, a 15,145 UPH. Como remos al cambiar las fórmulas ennpleadas en el cádculo ammenta el equivalente a L:PH, o lo que es lo mismo, en la fórmula antigua se contaba como clorofila a substancias que realmente no lo eran.

En la figura 15 se representa, igualmente, la relación clorofila total /UPH, cuya recta responde a la ecuación : [']' $=6580 \mathrm{mg}$ de clorofila +140 . En este caso $1 \mathrm{~g}$ de clorofila debe equivaler aproximadamente a 6,600 UPH. Exte rálol es sensiblemente mayor que los encontrados por otros autores, concretamente RuLey \& ('oxover (1956) que son de los autores que nhís se aproximan, dan valores inferiores en un $67 \%$, lo que parece resultar porque los extractos clorofílicos que emplearon en su trabajo no eran suficientemente puros.

Aunque los carotinojdes no se miden en gramos, dado que al aplicar las nuevas fómulas las unidades convencionales de medida se acercan bastante el gramo, hemos relacionado en la figura 16 la totalidad de los pigmentos expresado en $\mathrm{mg}$ en $\mathrm{ml}$ de extracto por $\mathrm{l}$ de agua filtrada con las correspondientes IIPH. I a ecuación de la recta resultante es: 


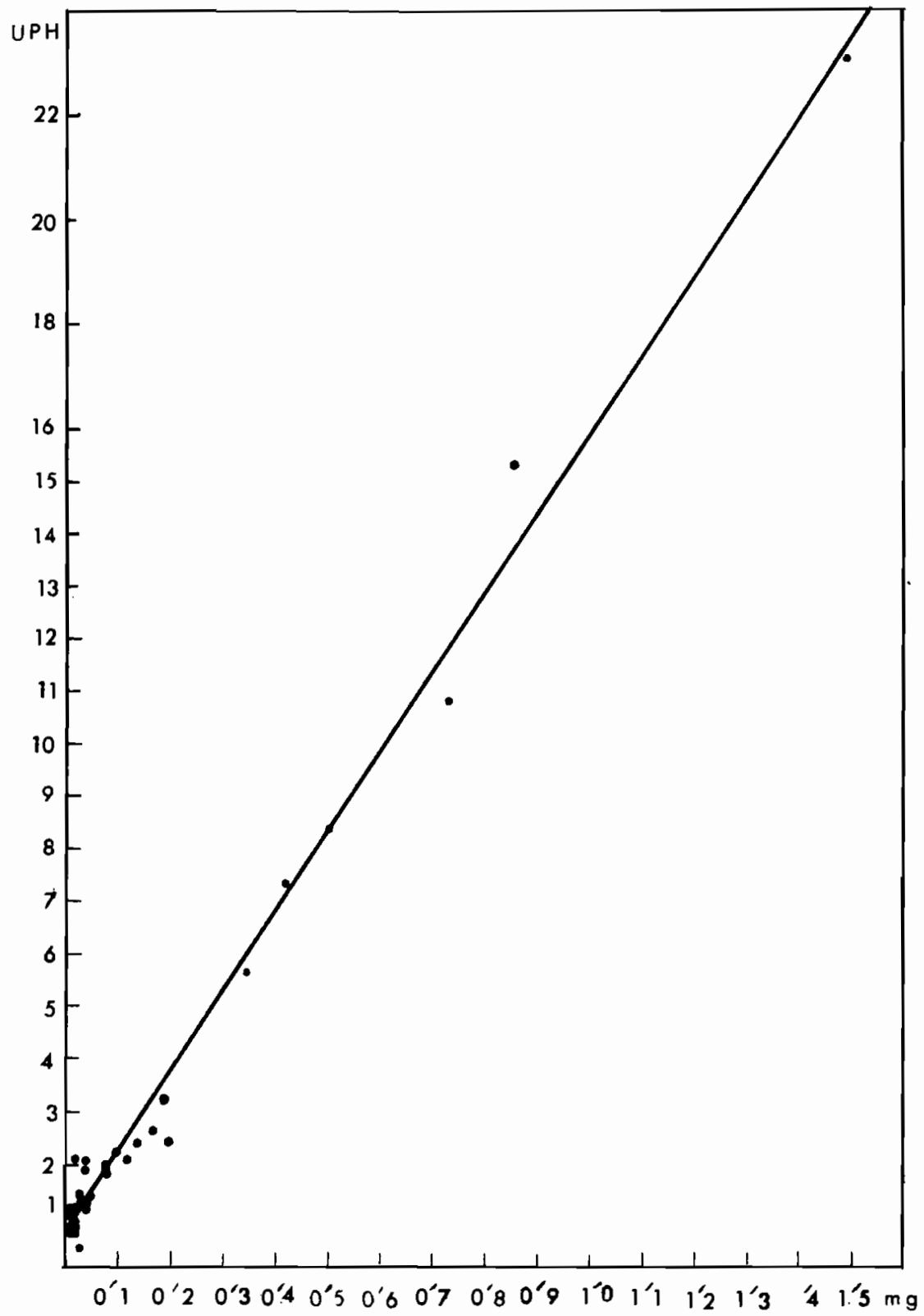

FIg. 14. - Relación entre la comentración d" clorofila a de una solución que en $1 \mathrm{ml}$ de extracto contione los pigmentos de $1 \mathrm{l}$ y el número de midades Harver por l de agua, calculadas mediante la fórmula UPH $=35.7 \mathrm{D}_{4,0}$. 'll la estación $\mathrm{B}$, de agosto de $190 \%$ a julio d. 19603 . La recta expresa la relación $\mathrm{CPH}=15145 \mathrm{mg}$ de clorofila $a+655$. I.a "lorotiln ha sido enlculadia a partir de las ecuaciomes at" Parsons y Strickland. 


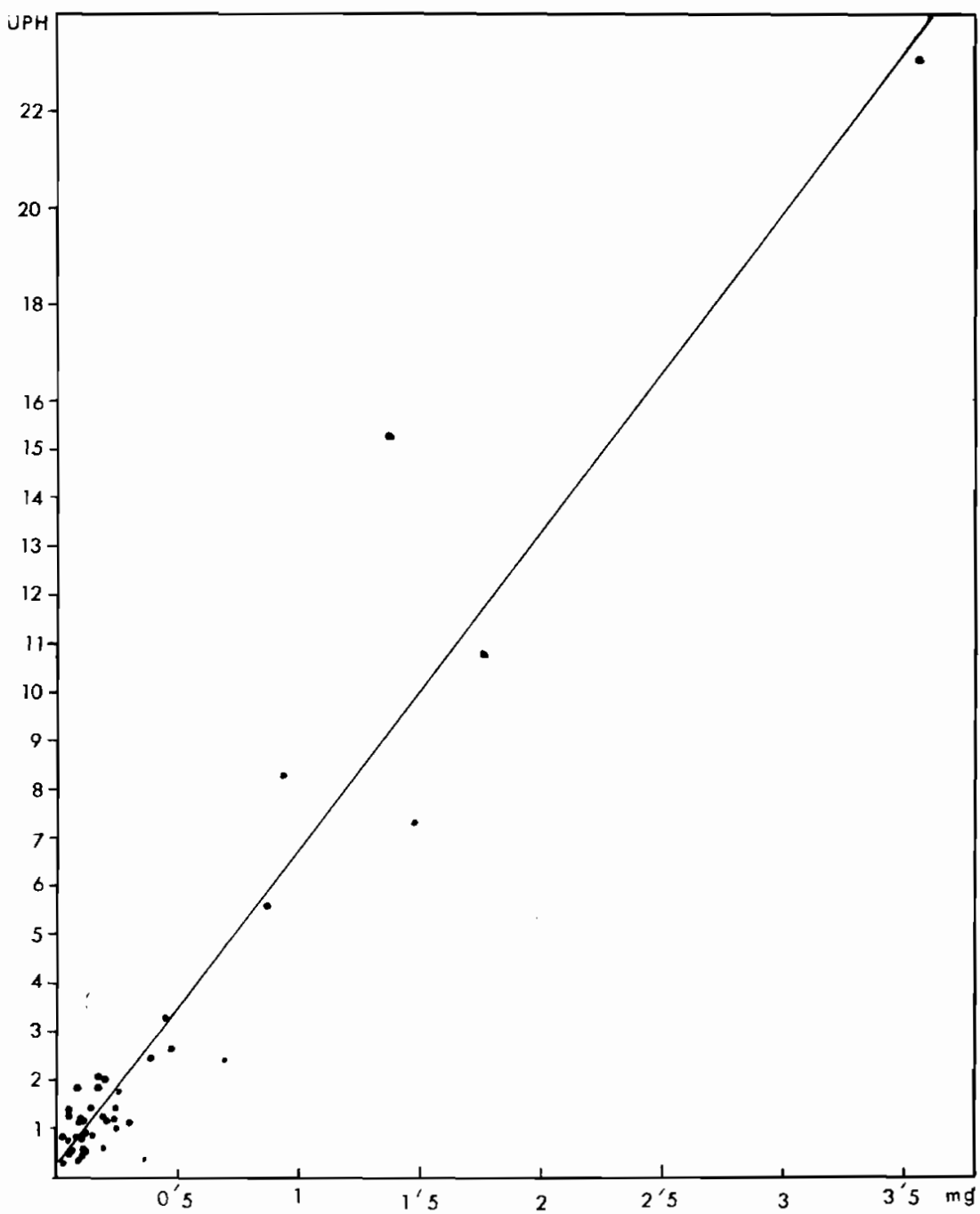

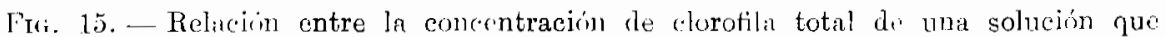
(n $1 \mathrm{ml}$ de extracto contieue Jos pigmentos de $1 \mathrm{l}$ y el múmoro de unidados Harvey por litro de agua. calculadas mediante la fórmula: $\mathrm{UPH}=35, \overline{\mathbf{T}} \mathrm{D}_{4: 3}$, de la cstación $\mathbf{B}$,

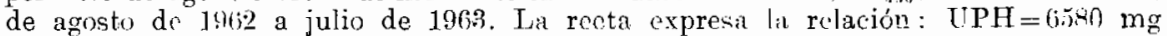
clorofila $\div$ 140. Ia chorofia ha sidn cilculada a putir d. las ecuaciones de Parsons $y$ Striclistand.

$\mathrm{UPH}=3750 \mathrm{mg}$ de pigmentos +405. No tenemos noticias de que algún autor haya relacionado el contenido total de pigmentos con las UPH, generalmente śstas se lian relacionado cun el peso seco, con cenizas o sin ellas, o el peso lunimedo del fitoplancton, o la clorofitit a o a lia clorofila 
total, pero grizí debido a dificultades técnicas la relación pigmentos totales/UPH, que nosotros sepamos, ha permanecido inédita. La relación que nosotros ofrecemos se da sólo a título de ensayo ya que, como indicamos en otro lugar, el agua filtrada no pasó a travís de la red de retención de zooplancton. Según ella 1 TPH equivale a $0.26 \mu g$ de pigmentos de plancton de origen vegetal.

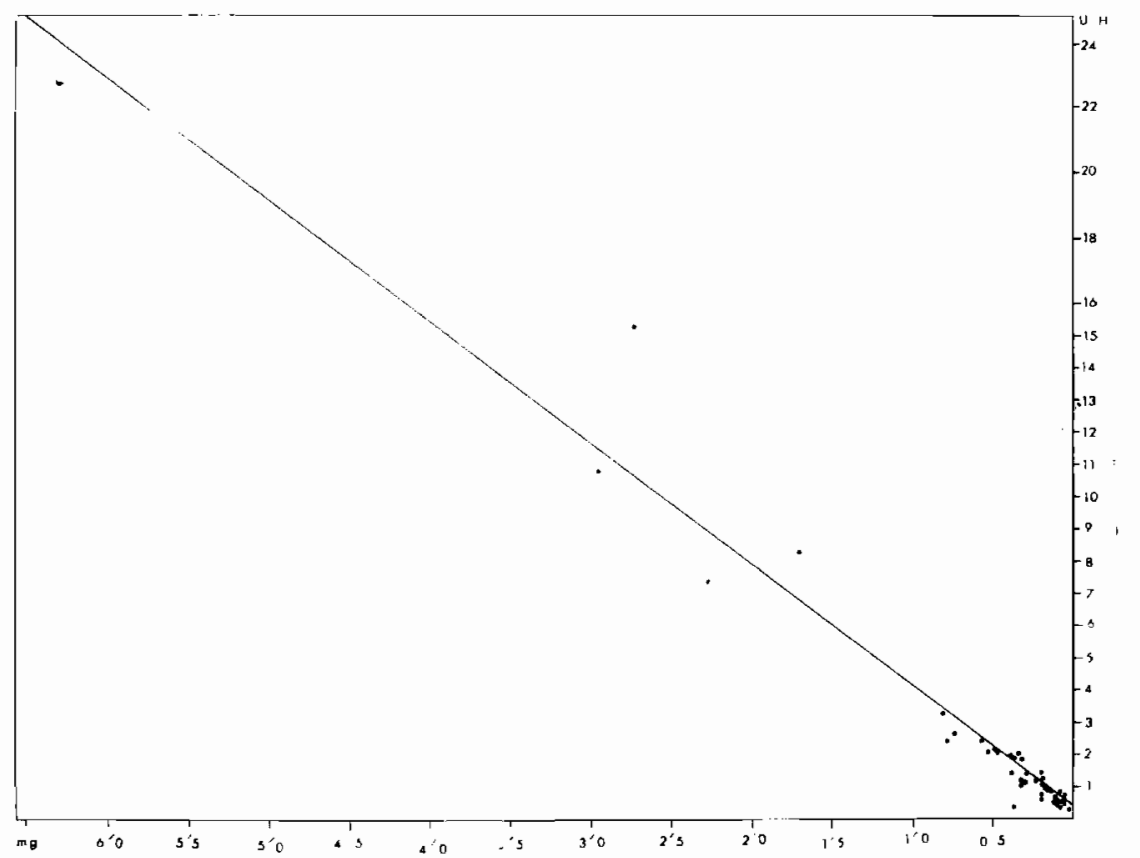

Fig. 16. - Relación untre la concentración de pigmentos (clorofilas $a, b$ y en mg $\because$ carotinoides en midades especificas) contenidas en $1 \mathrm{ml}$ de extracto $\mathrm{p}$ (m 1 de agua filtrada y el correspondiente número de unidades Harvey por litro, calculadas mediante la fórmula: $\mathrm{CPH}=85, \overline{7} \mathrm{D}_{430}$, de la estación $\mathrm{B}$, de agosto de 1962 a julio de 1963. La recta expresa la relación : $\mathrm{CPH}=3760 \mathrm{mg}$ de pigmentos +405 . Todos los pigmentos han sido calculados a partir de las ecuaciones de Parsons $y$ Strickland.

Taa distribución media de los pigmentos en nuestros extractos en el presente ciclo viene a ser la siguiente : $43 \%$ de carotinoides, $27 \%$ de clorofila $c, 20 \%$ de clorofila $a$ y $10 \%$ de clorofila $b$.

A continuación of recemos un chadro estadístico de las diferentes correlaciones; todas ellas presentan índices muy elevados, pero el mayor de todos es el correspondiente a la clorofila $a / \mathrm{UPH}$, cuya diferencia con el menor, clorofila total/UPH, aparece significativa. 
'I' I B L .I V I I

\begin{tabular}{|c|c|c|c|c|c|c|}
\hline & $\begin{array}{l}\text { INDICE DE } \\
\text { CORRELA - } \\
\text { CIÓN }\end{array}$ & $t$ & $r$ & $\begin{array}{c}\text { Desvia- } \\
\text { CIÓN } \\
\text { Tílicis }\end{array}$ & $\begin{array}{l}\text { LiMITES } \\
p=0,95\end{array}$ & $\begin{array}{r}\text { FidUCIARIOS } \\
p=0,98\end{array}$ \\
\hline Clorofilts $a / \mathrm{UPH}$ & 0,994 & i & 0,001 & 0,0019 & $0,990-0,997$ & $0,989-0,999$ \\
\hline Clorofila total/UPH & 0,957 & 23,327 & 0,001 & 0,012 & $0,934-0,980$ & $0,927-0,987$ \\
\hline Pigmentos/UPH & 0.978 & $m 3,7(1)-2$ & 11.0ला1 & 0,0046 & $0,967-0,980$ & $0.96: 3.0,993$ \\
\hline
\end{tabular}

\section{EL PRESEN'LE CICIOO ANIAT I SL RELACION CON LOS ANTERIORES}

El luecho más notable del presente ciclo es la aparición, en marzo, de una extraordinaria cantidad de fitoplancton que sigue a una gran pobreza en la primera quincena de febrero. En abril ruelve a obtenerse valores muy bajus, apareciendo un poco más elevados en mayo. Esta sucesión se corresponde, como hemos visto en los capítulos anteriores, con el curso de la concentración de fósforo inorgánico disuelto y, en cierto modo, con la invasión del área por aguas más densials procedentes de fondos inás profundos : posiblemente de origen septentrional.

La comparación con ciclos anteriores presenta, a causa de su heterogeneidad, notalsles dificultades, a pesar no obstante. de la ventaja que supone establecerla basándonos en la transformación de los datos de los últimos ciclos a LPH. Asi, la perioricidad en el presente ciclo suele ser de una rez por mes, mientras que en los anteriores de, al menos, una vez cada diez días, llegando a veces el valor mensual a ser el promedio de hasta diez determinaciones individuales. En estos casos la precisión de la duración e intensidad de la cosecha de fitoplancton tiene que ser forzosamente muclísimo mayor que ell el actual ciclo. Además, al intentar las comparaciones lógicainente lo haremos solamente ntiljzando los valores superficiales, ya que son los únicos de que disponemos en todos los ciclos anuales. El lugar de la toma de muestral tiene también su influencia y solamente en los últimos ciclos todas las muestras se tomaron en la misma estación.

Valores superiores a lals 7330 [iPH, encontradas el 15 de marzo, aparecen en los ciclos anteriores solamente en las siguientes fechas:

\begin{tabular}{|c|c|c|}
\hline (; febrero & $19 \div 51$ & $12+30$ \\
\hline () lebrero & 1951 & 16570 \\
\hline .) febrero & 1951 & 8270 \\
\hline 122 abril & 1951 & $8 \pm 00$ \\
\hline 1 mayo & 1.951 & $9 \pm 00$ y 9950 \\
\hline 20) febrero & 1953 & $8500^{\circ}$ \\
\hline
\end{tabular}




\begin{tabular}{|c|c|c|}
\hline 23 marzo & $195 \dot{3}$ & 11270 \\
\hline 25 febrero & 1955 & 7840 \\
\hline 28 febrero & 1955 & 13240 \\
\hline 2.) lebrero & 1957 & 15810 \\
\hline 15 noviembre & 1960 & 11140 \\
\hline
\end{tabular}

Por tanto solamente en los ciclos anuales 1950-1951, 1952-1953, 19541955 y 1956-1957 aparecen valores superiores al presente, jues no teneInos en cuenta el valor 11140 del 15 de nuviembre de 1960, pues ni en fechas anteriores o posteriores aparecen ralores próximos a éste, más aún, a 10 metros por debajo de esta muestra en la misma fecha la concentración era solamente de 1140 ITPH, por otra parte tampoco en ese mes se han encontrado munca valores tan elevados. Se debe tratal pues de un error o de una rara concentración superficial sin ningunal trascendencia.

TA B I A VII I.

\begin{tabular}{|c|c|c|c|c|c|c|}
\hline Estacionis & $1 .^{a}$ & $2 .^{\mathrm{a}}$ & $3 a^{a}$ & $\beta$ & $4 . a$ & $50^{2}$ \\
\hline $\begin{array}{l}\text { SITUACIÓN RESPECTO } \\
\text { A LA ESTACIÓN B }\end{array}$ & $\begin{array}{c}1 j, b \\
\text { MILLAS } \\
\text { AL SSO }\end{array}$ & $\begin{array}{c}8.1 \\
\text { MLLAS } \\
\text { AL SEE }\end{array}$ & $\begin{array}{c}3,4 \\
\text { MILLAS } \\
\text { 41. SSO }\end{array}$ & & $\begin{array}{c}10,8 \\
\text { MILLAS } \\
. \mathrm{F} \mathrm{F}\end{array}$ & $\begin{array}{r}18,2 \\
\text { MILLAS } \\
\text { AT. NNF }\end{array}$ \\
\hline Foxim & $100 \mathrm{~m}$ & $90 \mathrm{~m}$ & (i3) $\mathrm{m}$ & $75 \mathrm{~m}$ & 41) $\mathrm{m}$ & $62 \mathrm{~m}$ \\
\hline \multicolumn{7}{|l|}{ 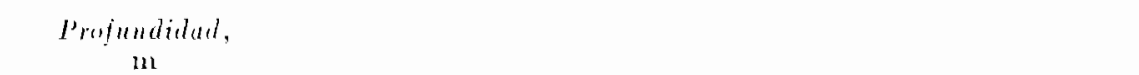 } \\
\hline 5 & & & & 38,19 & & \\
\hline 10 & & & 38.10 & 38,17 & 38,08 & 37.68 \\
\hline 25 & 38.24 & 39.17 & 38.13 & $38 \cdot 1$ & 38.28 & 38,01 \\
\hline 50 & $: 38,39$ & 38,31 & 38,39 & 38.25 & & 38,35 \\
\hline 75 & 38,35 & 38.37 & & 38,26 & & \\
\hline $10 n$ & $: 38.41$ & & & & & \\
\hline
\end{tabular}

Si la comparación la hacemos a través de medias mensuales, no aparece ninguna superiol a la registrada en marzo del presente ciclo, tomada como valor representativo mensual.

Lo que parece indudablemente claro es que, a partir del ciclo 1956 1957 no habia aparecido hasta el presente ningún otro con concentraciones superiores de fitoplancton y que en el actual parece que se vuelve a las condiciones de producción existentes en los primeros ciclos del estudio, las cuales de año en año habian venido descendiendo sistemáticamente hasta alcanzar valores que no permitían diferenciar el semiciclo productivo del semiciclo estival. También los ciclos 1951-1.952, 19531954 y 1955-1956 fueron menos productivos que el actual. 
En cuanto a los cuatro restantes es difícil establecer cuál de ellos aventajó al presente en la producción, pero si juzgamos a través de la salinidad y concentración de fósforo inorgínico disuelto, el ciclo 1956 1957 tuvo que ser inferior, no sólo porque la salinidad fue algo más baja que en el presente, sino porque la concentración de fósforo fue notitblemente inferior. Tenemos la fortuna de poseer datos de salinidad del ciclo 1950-1951 (Institu'To Español de Ockanogikafía, 1957) justamente en la misma fecha (15 de marzo) en la que nosotros en el ciclo actual encontramos el máximo de producción. Oflecemos a continuación (tabla VIII) los datos referentes a las cinco estaciones más próximas a la nuestra junto con los referentes a ella misma.

De aquí se desprende que, el volumen de l'enloción de aguas profundas ha debido ser de características muy similares en ambos ciclos por estas fechas y las diferencias que se observan pueden ser debidas a una mayor evolución en el presente ciclo que no sabemos si prosiguió en el de 1950-1951, aunque a través de las determinaciones de fitoplancton suponemos que no, sin perjuicio de que un mes más tarde se volvieran a producir. Además en el mes de febrero se produjo otra abundante co- . secha que no ha aparecido en el presente ciclo.

Nada podemos decir de los dos ciclos restintes, ya que solanente poseemos los datos de producción de fitoplancton, pero al menos parece ser que el ciclo 1952-1953 fue más prolongado y quizá también el ciclo 1954-1955.

\section{SL M Y ARY}

Hydrography and phytoplankton of the coust of Castellin (IVist Mediterrunean) from August 1962 to July 19633 . - Dit i of temperature, salinity, density, organic phosphate content, surface currents. Secchi dise reading and wind intensity in relation to upwclling are reported: these dita ile plotted in several figures and the. numeric values listed in several tables. Also plant pigment are given. Previous working methods have not been modified, but the study is linited to station b $\left(39^{\circ} 55^{\prime} 30^{\prime \prime} \mathrm{N}, 0^{\circ} 21^{\prime} 30^{\prime \prime} \mathrm{E}\right)$.

The progressive reduction of inorganic phosphate content of the wattre, observed during the last ycars, his reversed its trend in present annual aycle. The possible canse of this fellomenon is tentatively sought in an occupancy of the shelf by dense water coming up from the bottom, probably formed in the Gulf of Lion. and perhaps helped in its uprard movement hy third quadrant winds. This invasion caused a lowering of the temperature that reached values so far unknown, and an increase in the salinity, density and inorganic phospliate content till to ralues never recorded. Effects of this invasion on the biologieal production were notable only in March, the production in this month amounted to the production of the rest of the year; under $40 \mathrm{~m}$, at the end of May, the productivity was also notable. The production

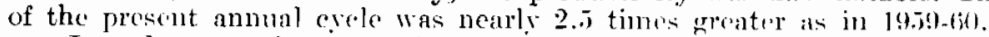

In order to malie comparisons. dita of plant pioment are calculated using both the Richards and 'Themplem formulis and the mer rxpresions of Parsons and Strickland. The last ones give valures lower than the first. With the values ealculated through the Parsons and Striclian formulas the relations betwen chlorophyll $a$, 
whole chlorophyll, and whole pignnents on the one hand, and Harvey units on tho other are computed. The three correlations are fair, but the first one is the best. The production of these waters during all the annual cycles studied is compared and it is concluded that, if the present anmual cycle is not the best, it may b. included among the four better.

\section{B I B L I O G R A FIA}

Bougrs, P. - 195̃ . Contribution ì la connaissance des eourants superticiels dans le nord-ouest de la Méditerranée occidentale. Comm. int. Érplor. sci. Méditcranée, Rapp. et P. I., vol. 14 11. s. : $67-84$.

Funnestin, J. - 1964). Hydrologie de la Méditerranée occidentale (golfe du Lion, Mer Catalane, Mer d'Alboran, Corse orientale) 14 juin-20 juillet 1957. Rev. Trav. Inst. Pêches marit., 24 (1): 5-119.

Funnestin, J. \& Ch. Alliin. - 1962. Hydrologie de la Méditerranée occidentale au nord du $42^{\circ}$ parallèle en automne 1958 (Campagne du navire «President-'Théodore-Tissier» du 27 septembre au 18 octobre 1958). Rev. Trav. Inst. Pêches marit., 26 (2) : 113-163.

Herrera, J. \& R. Margalef. - 195T. Hidrografía y fitoplancton de las costas de Castellón, de julio de 1956 a junio de 1957. Inv. Pesq., 10: 17-14.

- 1961. Hidrografia y fitoplancton de las costas de Castellón, de julio de 1958 a junio de 1959. Inv. Pesq., 20: 17-63.

- 1963. Hidrografía y fitoplancton de la costa comprendida entre Castellón y la desembocadura del Ebro, de julio de 1960 a junio de 1961. Inv. Pesq., 24: 33-1]2.

Instituto Éspañol de Oceanoghafia. - 195i. Campanas del «Aalien» en ol Medíterráneo español y en las costas Noroeste y Cantábrica de España en el año 1951. Bol. Inst. H'sp. Occan., 83 : 1-38.

Margaley, R. - 19hiu. Valeur indicatrice de la composition des pigments du phytoplancton sur la productivité, composition taxonomique et propiétés dynamiques des populations. Rapp. Proc. Torb. reun. C.I.E.S.M.M., 15 (2) : 227-281.

- 1963. El ecosistema pelágico de un área costera del Mediterráneo occidental. Mem. Real Acad. Cirne. Art.. Barceloma 25. 1.

Margales, R. \& J. Herrera. - 1964. Hidrografía $y$ fitoplancton de la costa comprendida contre Castellón y la destmbocadura del Ebro, de julio de 1 thit a juniu de 1962. Inv. Pesq., $26: 49-90$.

Margalef, R.. J. Herrera \& E. Arlas. - 1959. Hidrogratía y fitoplancton de las costas de Castellón, de julio de 1957 a junio de 1958. Inv. Pesq., 15:3-38.

Muñoz, F. - 1964. Datos climáticos del puerto de Castellón, en 1963. Inv. Pesq., $27: 147-158$.

Parsons, T. R. \& J. D. Strickland. - 1963. Discussion of spectrophotometric determination of marine-plant pigments, with revised equations for ascertaining chlorophylls and carotenoids. $J, .1 / a r$. Res., $21: 155-163$.

Rirhards, F. A. \& T. Thompson. - 1952. The estimation and characterization of planliton populations by pigments analysis. II. A spectrophotometric method for the estimation of planliton pigments. I. Mar. Res., $11: 156$ - 172 .

RiLeY, G. A. A S. A. M. M. CoNor LR. - 1956. Oceanography of Long Island Sound, 1952-1954. III. Chemical Oceanographs. Bull. Bingham Occanogr. Coll., 15: $\div \overline{7} .61$.

Rither, J. H. \& C. S. Yentsen. - 1957. The estimation of phytoplankton production in the oceall from chlorophyll and ligh data. Limnol. and Oceanogr., 2: 281-28ti.

Sute, P. \& F. Vives. - 1958. Estudio do las corrientes superficiales del Mediterráneo occidental. Rapp. Proc. Verb. reun. C.I.E.S.M.M., 14: 53-65.

Tohernia, P. - 1956. Contribution à l'etude hrdrologique de la Méditurrance Ocecidentale. Bull. Inform.. \& (9) : $427-454$.

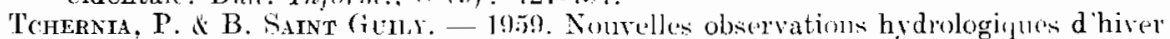
en Méditerranéu oceidentale. ('ah. Ocednogr. C.O.L.C., 11 (i) : 499-i)t2.

Yosms. K. - 1955. Constal upwelling off the California coast and its effects on

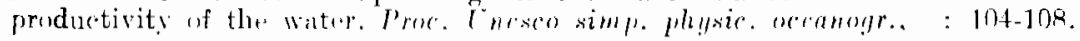


$\begin{array}{lllllllllllll}A & P & E & N & D & I & C & E\end{array}$

$=$

\begin{tabular}{|c|c|c|c|c|c|c|c|c|c|c|c|c|c|c|c|c|c|c|c|}
\hline \multirow[b]{3}{*}{ 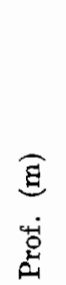 } & \multirow[b]{3}{*}{$\begin{array}{l}0 \\
0 \\
\dot{0} \\
0 \\
0\end{array}$} & \multirow[b]{3}{*}{ 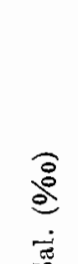 } & \multirow[b]{3}{*}{$E$} & \multirow[b]{3}{*}{ 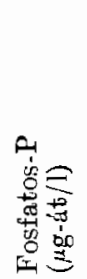 } & \multirow[b]{3}{*}{$\stackrel{0}{0}$} & \multirow[b]{3}{*}{$\overbrace{}^{8}$} & \multirow[b]{3}{*}{$\underbrace{\theta_{0}^{\circ}}_{0}$} & \multicolumn{5}{|c|}{ Pigmentos } & \multirow[b]{3}{*}{$\frac{5}{5}$} & \multirow{2}{*}{\multicolumn{6}{|c|}{ Fitoplancton en núm. céls. por litro (*) }} \\
\hline & & & & & & & & & & & 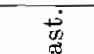 & & & & & & & & \\
\hline & & & & & & & & $\begin{array}{l}0 \\
0 \\
010 \\
0 \\
0 \\
0 \\
0\end{array}$ & $\frac{\left(\mathrm{mg} / \mathrm{m}^{3}\right.}{\frac{\pi}{c}}$ & 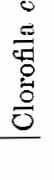 & 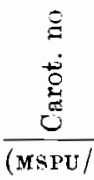 & 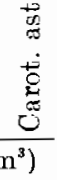 & & 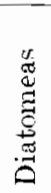 & 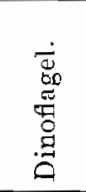 & $\begin{array}{l}+4 \\
\stackrel{0}{0} \\
\stackrel{0}{0} \\
\stackrel{0}{8}\end{array}$ & 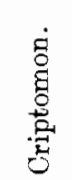 & 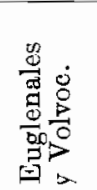 & \\
\hline
\end{tabular}

23-VIII-62.945-1283 GMT. T. aire, $27,3^{\circ} \mathrm{C}$. Pres. atm., $764 \mathrm{~mm}$. D. Secchi, $31 \mathrm{~m}$. Corr. sup. sW, $12,4 \mathrm{~cm} / \mathrm{seg}$. Mar ealma.

\begin{tabular}{|c|c|c|c|c|c|c|c|c|c|c|c|c|c|c|c|}
\hline $\begin{array}{l}26,1 \\
26,2\end{array}$ & $\begin{array}{l}37,67 \\
37,59\end{array}$ & $\begin{array}{l}25,05 \\
24,97\end{array}$ & & 0,017 & 0,003 & 5,7 & 0,04 & 0,05 & 0,30 & 0,06 & 640 & 160 & 1000 & 500 & $\begin{array}{l}2830 \\
8330\end{array}$ \\
\hline 26,2 & 37,59 & 24.47 & $0 .(19)$ & $(1),(1) 22$ & $(0.00)$ & 11,0 & 0.01 & 0,01 & 0,30 & $0,0.4$ & .540 & itis & $\begin{array}{l}+320 \\
2665\end{array}$ & $\begin{array}{l}000 \\
1160\end{array}$ & $T \cos 0$ \\
\hline 20,9 & & & & & & & & & & & & & & & \\
\hline 18,2 & 37,50 & 27.15 & 0,04 & $10,02: 3$ & 0,0022 & $11 . \pi$ & 0,01 & 0,022 & 0,20 & 0.03 & 500 & & & & \\
\hline $\begin{array}{l}10,6 \\
16,6\end{array}$ & 37,47 & 27.52 & 0,16 & & & & & & & & & $1 t: t j$ & 2880 & 500 & 7320 \\
\hline 15.8 & & & & & & & & & & & & & & & \\
\hline $\begin{array}{l}15,1 \\
14,7\end{array}$ & & & & 0,1126 & 0,005 & 5.2 & 0,05 & 0,00 & 0,22 & 11,07 & 660 & & & & \\
\hline $\begin{array}{l}14,7 \\
14,0\end{array}$ & 37,97 & 28.30 & 0,04 & & & & & & & & & 667 & 21650 & 383 & 8332 \\
\hline $\begin{array}{l}13,8 \\
13,7\end{array}$ & & & & 0,028 & 0.0182 & 11,5 & 0.01 & & 0,49 & 0,04 & 620 & & & & \\
\hline 13,3 & & & & & & & & & & & & 1330 & 1. 000 & 333 & 4.162 \\
\hline
\end{tabular}

$\begin{array}{llllll}75 & 13,1 & 38,03 & 28,74 & 0,05\end{array}$

10-X-62. 1015-12li) GMT. T. aire, $24^{\circ} \mathrm{C}$. Pres. atm., $762 \mathrm{~mm}$. D. Secchi, $34 \mathrm{~m}$. Corr. sup. li. 4, (i cm/seg. Mar rizada.

\begin{tabular}{|c|c|c|c|c|c|c|c|c|c|c|c|c|c|c|c|c|c|}
\hline 0 & & & & 0,081 & 0,005 & 6,2 & 0,07 & 0,07 & 0,45 & 0,01 & 0,04 & 1180 & & & & & \\
\hline 5 & 23,4 & $\begin{array}{r}37,42 \\
\quad 37\end{array}$ & $\begin{array}{r}25,66 \\
25\end{array}$ & & & & & & & & & & $83: 3$ & 8650 & 1000 & 500 & 19150 \\
\hline 15 & $\begin{array}{l}22,4 \\
22,9\end{array}$ & & 20,00 & 0,051 & 0,006 & 8,5 & 0,06 & 0,05 & 0,49 & 0,00 & 0,00 & 1430 & $83 \pi$ & $+0,00$ & 6060 & & 10680 \\
\hline 20 & 22,7 & 37,70 & 26,07 & 0,034 & 0,004 & 8,5 & 0,04 & 0,00 & 0,28 & & 0,04 & 830 & 500 & 4160 & 335 & & 7160 \\
\hline
\end{tabular}


APENDICE (Continuacion.)

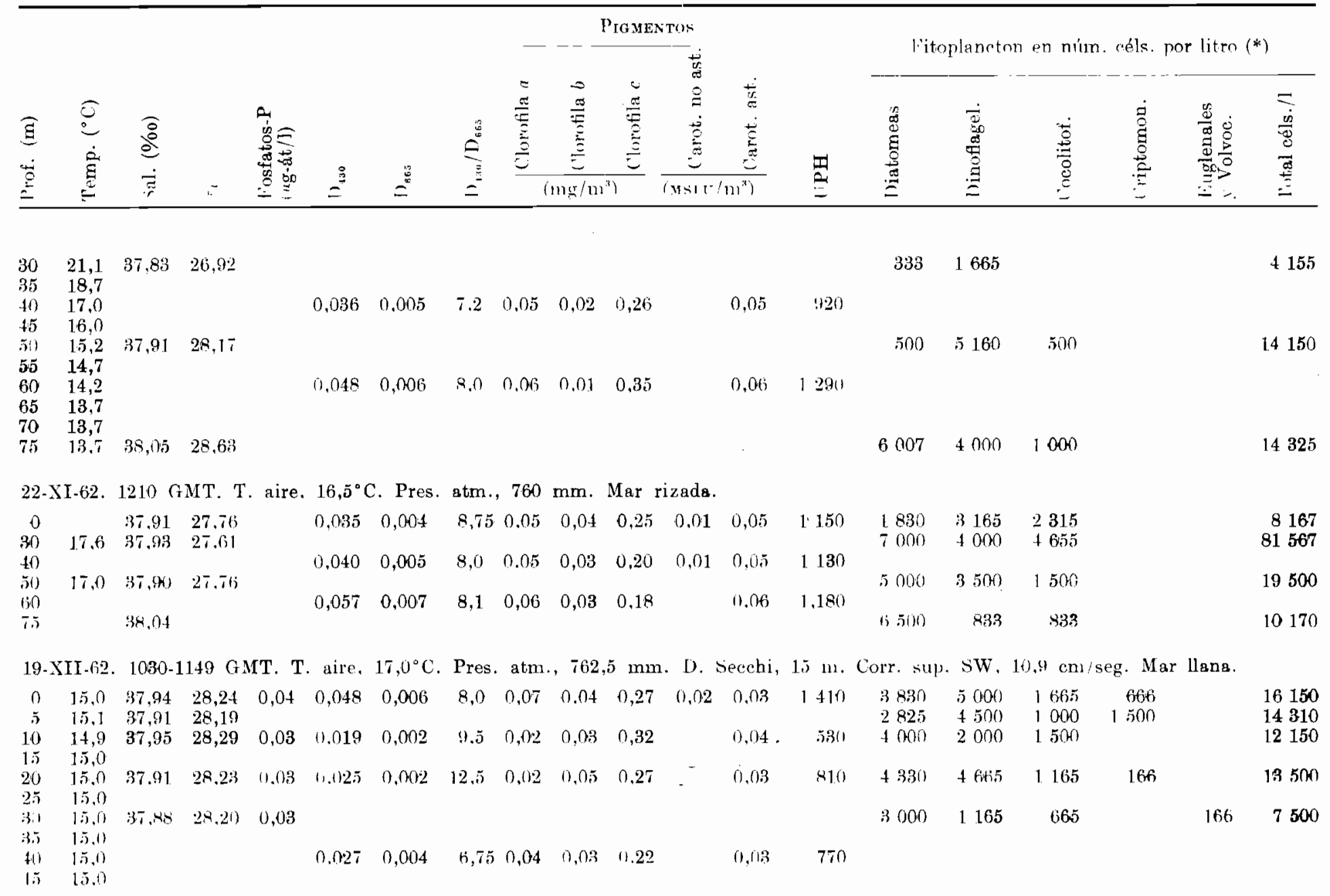


6-II.63. $1110-1155$ GMT. T. aire, $13,0^{\circ} \mathrm{C}$. Pres. atnı, $759 \mathrm{~mm}$ D. Secchi, $13 \mathrm{~m}$. Mar de fondu.

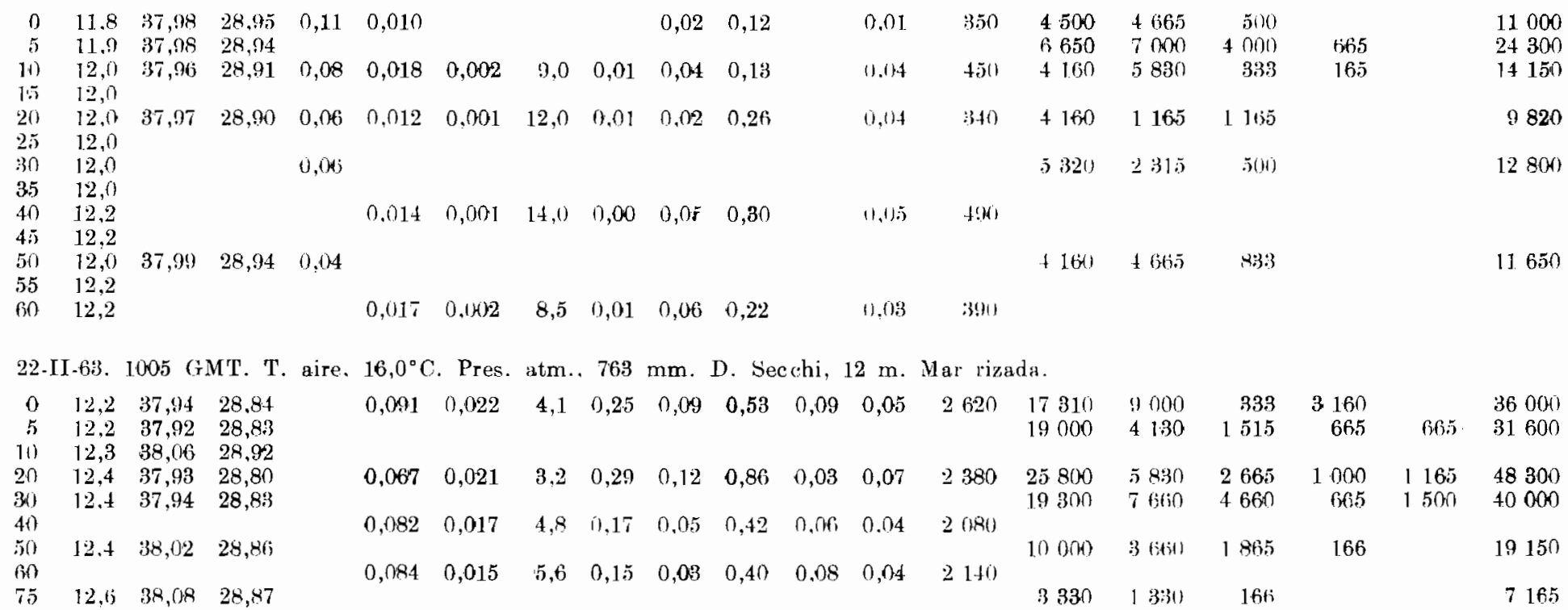

15-III-63. 1040-1210 GMT, T. aire, $15^{\circ} \mathrm{C}$, Pres, atm., $763 \mathrm{~mm}$. D. Secchi, $7,30 \mathrm{in}$. ('orr. sup. N, 11,5 cm/seg. Miar de fondo.

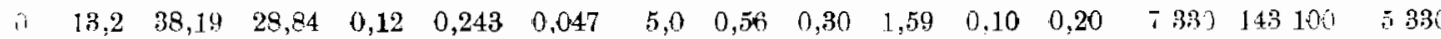

$4000 \quad 4000 \quad 268000$

i) $13,0 \quad 38,19 \quad 28,88$

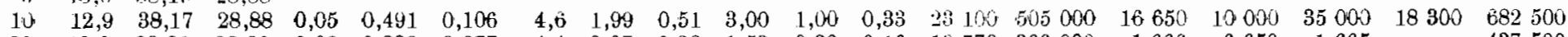

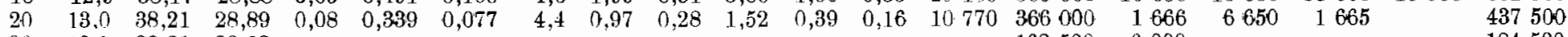

$\begin{array}{llll}162500 & 6000 & 184500\end{array}$

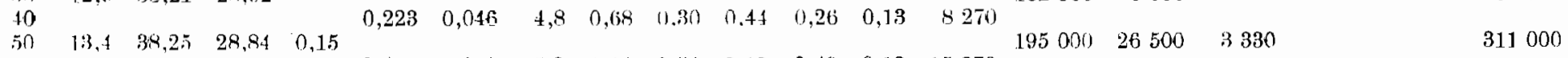

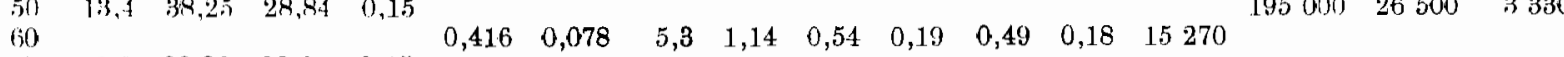

$72,9 \quad 38,26 \quad 28,97 \quad 0,15$ 
IPENDICE (Continuación.)

I.itoplancton en núin. céls. por litro (*) PIGMENTus

3.IV-63. 1045 GMT. T. aire, 17,7 ${ }^{\circ} \mathrm{C}$. Pres. atm., 761,7 mm. D. Seccbi, $15 \mathrm{~m}$. Corr. sup. N, $7.1 \mathrm{~cm} / \mathrm{seg}$. Mar de fondo.

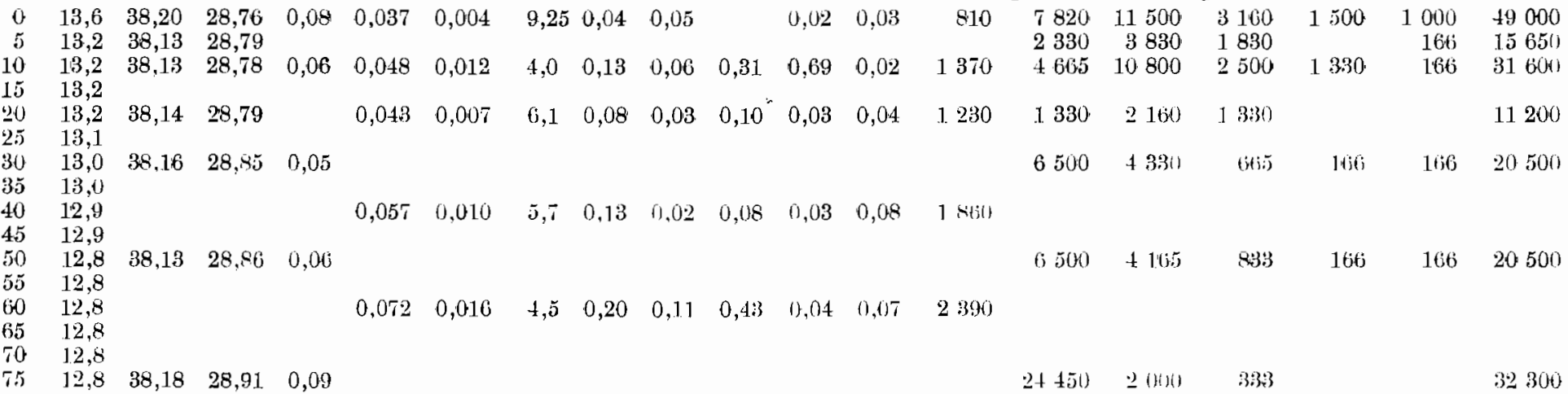

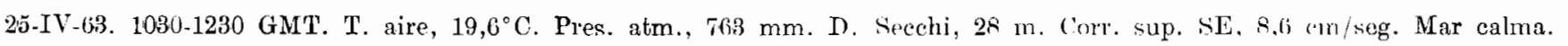

\begin{tabular}{|c|c|c|c|c|c|c|c|c|c|c|c|c|c|c|c|c|c|c|}
\hline $\begin{array}{l}15,4 \\
15,1\end{array}$ & $\begin{array}{l}37,77 \\
37,77\end{array}$ & $\begin{array}{l}28,03 \\
28,10\end{array}$ & 0,06 & 0,027 & 0,004 & 6,75 & 0,04 & 0,05 & 0,32 & & 0.06 & 870 & 2330 & 6330 & 500 & $\begin{array}{r}166 \\
0.00\end{array}$ & $\begin{array}{r}500 \\
1833\end{array}$ & $\begin{array}{l}31.000 \\
29600\end{array}$ \\
\hline $\begin{array}{l}15,0 \\
15,0\end{array}$ & 37,97 & 28,14 & $0,0 \pm$ & 0,020 & 0,003 & 6,7 & 0,04 & 0,03 & 0,20 & & $0,0.33$ & 710 & & & & & & \\
\hline $\begin{array}{l}14,8 \\
14.1\end{array}$ & 37,83 & 28,22 & 0,03 & 0,069 & 0,005 & 13,8 & 0,05 & 0.06 & 0,35 & 0,13 & $0,0 \overline{3}$ & 2080 & 166 & 2500 & 266 & & 2060 & 161.150 \\
\hline 12,8 & $37,9 \mathrm{~K}$ & 28,76 & & & & & & & & & & & 500 & 5830 & & & 266 & 13500 \\
\hline & 37,95 & & 0,03 & & 0,000 & & $0,0 i$ & , Uכ & ,63 & & , & & 166 & $231 \bar{t}$ & 166 & 1665 & & 9330 \\
\hline 12,7 & 38,28 & 29,00 & 0,09 & 0,000 & 0.011 & 5,7 & 0,13 & 0,188 & 0,32 & 0,08 & 0,06 & & 383 & 1660 & & & & 6160 \\
\hline
\end{tabular}




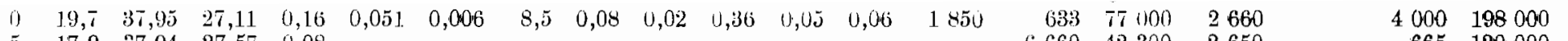

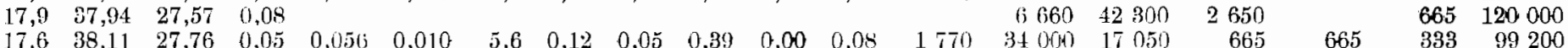

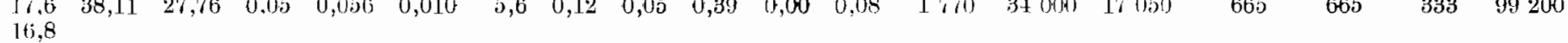

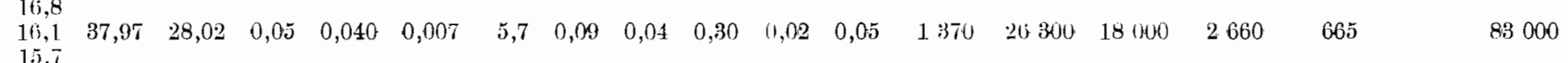

$14 . \overline{1} \quad 38,04 \quad 28,38 \quad 0,04$

$45300 \quad 12000 \quad 2860$

91500

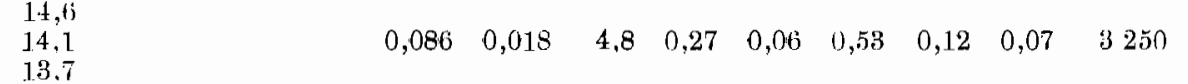

$13,3 \quad 38,1 / \quad 28,84 \quad 0,02$

$134700 \quad 12300 \quad 1660$

333

202500

13,0

$\begin{array}{lllllllll}0,174 & 0,037 & 4,7 & 0,48 & 0,12 & 0,86 & 0,19 & 0,10 & 5630\end{array}$

(2.8 $\quad 3 \times, 21 \quad 28,94 \quad 0,11$

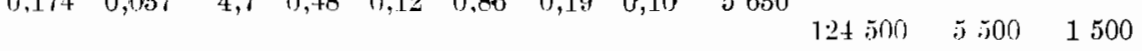

1.64000

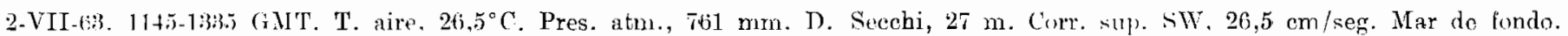

\begin{tabular}{|c|c|c|c|c|c|c|c|c|c|c|c|c|c|c|c|}
\hline $23 . i i$ & $\begin{array}{l}37.7 .5 \\
37, \pi 1\end{array}$ & $2: 5.86$ & $\begin{array}{l}0,06 \\
0,06\end{array}$ & 0,029 & 0,003 & 9,7 & 0,08 & 0,05 & 0,46 & 0,04 & $1: 010$ & 1.66 & 7500 & 666 & 15650 \\
\hline $\begin{array}{l}22, ! ! \\
22,1\end{array}$ & 38,015 & 26,29 & 0,03 & 0,028 & 0,004 & $\overline{7}, 0$ & 0,04 & 0,03 & 0,22 & 0,06 & 1090 & 500 & 7150 & 833 & 16300 \\
\hline
\end{tabular}

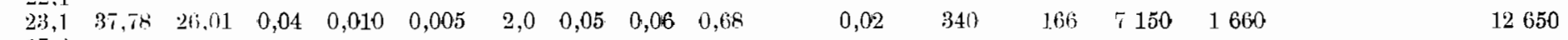

$16,2 \quad 38,033 \quad 28,(1) 4 \quad 0,04$

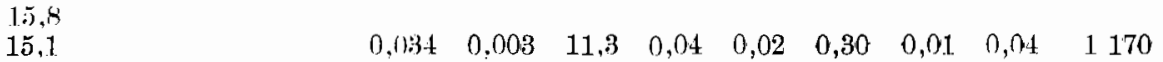

15,9

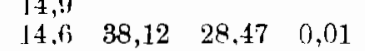

$833 \quad 5660 \quad 1500$

16150

$\begin{array}{lllllllll}1+, 4 & 0,0183 & 0,006 & 10,5 & 0,07 & 0,06 & 0,37 & 0,10 & 2030\end{array}$

14,4

14,4

$\begin{array}{lllll}5 & 13,3 & 38.15 & 28.57 & 0,09\end{array}$

26-VII-63. 1045-1140 GMT. 'I' aire, 26,5 C. Pres, atm., $762 \mathrm{~mm}$. D. Secehi, 21 111. Corr. sup. SW, $20 \mathrm{~cm} / \mathrm{seg}$. Mar calma.

\begin{tabular}{rcccc}
0 & 26,3 & 37,65 & 24,95 & 0,10 \\
5 & \multicolumn{3}{c}{37,64} \\
10 & 26,1 & 37,56 & 24,95 & 0,02 \\
20 & 23,4 & 37,53 & 25,74 & 0,03 \\
30 & 15,5 & 37,66 & 27,94 & 0,04 \\
50 & 15,3 & 38,12 & 28,33 & 0,03 \\
75 & 15,2 & 38,13 & 28,36 & 0,04
\end{tabular}

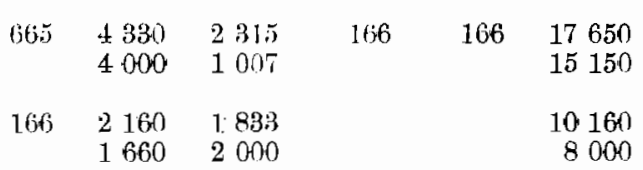

(*) La diferencia entre la suma de los grupos especificos y el tutal corresponde principalmunte a pe(puenios tlagelados, ('u su mayor parte crisomodales. 